\title{
Stress-constrained topology optimization considering uniform manufacturing uncertainties
}

\author{
Assis da Silva, Gustavo; Teófilo Becka, André ; Sigmund, Ole
}

Published in:

Computer Methods in Applied Mechanics and Engineering

Link to article, DOI:

10.1016/j.cma.2018.10.020

Publication date:

2019

Document Version

Peer reviewed version

Link back to DTU Orbit

Citation (APA):

Assis da Silva, G., Teófilo Becka, A., \& Sigmund, O. (2019). Stress-constrained topology optimization considering uniform manufacturing uncertainties. Computer Methods in Applied Mechanics and Engineering, 344, 512-537. https://doi.org/10.1016/j.cma.2018.10.020

\section{General rights}

Copyright and moral rights for the publications made accessible in the public portal are retained by the authors and/or other copyright owners and it is a condition of accessing publications that users recognise and abide by the legal requirements associated with these rights.

- Users may download and print one copy of any publication from the public portal for the purpose of private study or research.

- You may not further distribute the material or use it for any profit-making activity or commercial gain

- You may freely distribute the URL identifying the publication in the public portal 


\title{
Stress-constrained topology optimization considering uniform manufacturing uncertainties
}

\author{
Gustavo Assis da Silva ${ }^{\mathrm{a}}$, André Teófilo Beck ${ }^{\mathrm{a}}$, Ole Sigmund ${ }^{\mathrm{b}}$ \\ ${ }^{a}$ Department of Structural Engineering, São Carlos School of Engineering, University of São Paulo, 13.566-590, São Carlos, SP, Brazil \\ ${ }^{b}$ Department of Mechanical Engineering, Solid Mechanics, Technical University of Denmark, Nils Koppels Alle, B. 404,2800 Kgs. Lyngby, \\ Denmark
}

\begin{abstract}
This paper proposes a robust design approach, based on eroded, intermediate and dilated projections, to handle uniform manufacturing uncertainties in stress-constrained topology optimization. In addition, a simple scheme is proposed to increase accuracy of stress evaluation at jagged edges, based on limiting sharpness of the projections to intentionally allow a thin layer of intermediate material between solid and void phases. A reference problem is analyzed through voxel-based finite element models, demonstrating that, in association with a proper choice of stiffness and stress interpolation functions, the proposed scheme can ensure consistent stress magnitude and smooth stress behavior for uniform boundary variation. Optimization problems are solved and post-processing with body-fitted meshes is performed over optimized solutions, demonstrating that: 1) stresses evaluated with voxel-based meshes containing thin soft transition boundaries are consistent with stresses evaluated with body-fitted meshes; and 2) optimized structures are robust with respect to uniform boundary variations.
\end{abstract}

Keywords: Topology optimization, Stress constraints, Manufacturing uncertainties, Robust design

\section{Introduction}

It is nowadays widely acknowledged that consideration of stress constraints in design of continuum structures is sometimes necessary. Since the pioneering work of Duysinx and Bendsøe [1], stress-based topology optimization of continuum structures has become largely widespread in the literature (see, e.g., references [1-31]), due to its large applicability in structural design. However, although remarkable achievements have been obtained so far, stress-based design approaches have been mostly employed to solve deterministic problems, which are formulated by assuming perfectly known properties, parameters and operating conditions.

Since most engineering problems are subjected to some source of uncertainty [32, 33], the development of formulations that can properly handle stress-based design problems under uncertainty becomes very important. A comprehensive literature review revealed some papers proposing stress-based topology optimization formulations able to take uncertainties in applied loads and/or material properties into account [25-31], by employing either reliability-based [34] or robust design $[32,35]$ approaches. However, to the authors' best knowledge, there is no work in the literature addressing the stress-based design problem considering manufacturing uncertainties.

\footnotetext{
Email addresses: gustavoassisdasilva@gmail.com (Gustavo Assis da Silva), atbeck@sc.usp.br (André Teófilo Beck), sigmund@mek.dtu.dk (Ole Sigmund)
} 
Hence, this work aims at employing the non-probabilistic robust formulation, proposed in [36, 37], based on eroded, intermediate and dilated projections, to handle uniform manufacturing uncertainties in stress-constrained topology optimization. Although the three-field robust approach employed herein can be seen as an established tool for obtaining robust designs, investigation of its applicability over stress-based design is still required.

The main objectives/contributions of this work are:

1. Proposing a robust formulation, based on $[36,37]$, to handle the problem of volume minimization with local stress constraints, considering uniform manufacturing uncertainties;

2. Demonstrating that, under reasonable assumptions regarding model parameters, the phenomenon of artificial stress concentrations at jagged boundaries [17] can be alleviated, and stress accuracy at the interface between solid and void regions can be ensured;

3. Demonstrating, through proper post-processing, that optimized structures are indeed manufacturing tolerant designs that satisfy stress constraints.

The paper is organized as follows: the stress-constrained formulations (both deterministic and robust) and optimization procedure are presented in section 2; the problem of stress evaluation at jagged boundaries is addressed in section 3; optimization examples and proper validation are shown in section 4; complementary numerical examples are shown in section 5; and concluding remarks are given in section 6. Sensitivity analysis is given in the appendix.

\section{Stress-constrained continuum topology optimization}

In this paper, the volume minimization problem subjected to local stress constraints is addressed. The traditional density approach [38] is employed: the structural problem is solved with the displacement-based finite element method for linear elasticity under static loads [39] and in addition, each finite element $e$ is associated with a relative density $\bar{\rho}_{e}$ varying from 0 (which represents void) to 1 (which represents solid). Relative densities $\bar{\rho}$ are related to design variables $\rho$ through density filtering with threshold projection [37]. The difference between deterministic and robust formulations is in the simultaneous consideration of three distinct relative density fields in the robust case: dilated $\overline{\boldsymbol{\rho}}^{(d)}$, intermediate $\overline{\boldsymbol{\rho}}^{(i)}$ and eroded $\overline{\boldsymbol{\rho}}^{(e)}$; instead of only one field $\overline{\boldsymbol{\rho}}$ as in the deterministic case. Next subsections are devoted to present the traditional deterministic stress-constrained problem of volume minimization (subsection 2.1), the proposed robust problem (subsection 2.2), the threshold projection scheme (subsection 2.3), and the proposed solution procedure (subsection 2.4). 


\subsection{Deterministic formulation}

Considering the von Mises failure criterion, one can write the deterministic stress-constrained volume minimization problem in discrete form as

$$
\begin{array}{lll}
\underset{\boldsymbol{\rho}}{\operatorname{Min} .} & V(\overline{\boldsymbol{\rho}})=\sum_{e=1}^{N_{e}} V_{e} \bar{\rho}_{e} & \\
& & \\
\text { s. t. } & \frac{\sigma_{e q}^{(k)}(\overline{\boldsymbol{\rho}})}{\sigma_{y}}-1 \leqslant 0 & k=1,2, \ldots, N_{k}, \\
& \mathbf{K}(\overline{\boldsymbol{\rho}}) \mathbf{U}(\overline{\boldsymbol{\rho}})=\mathbf{F} & \\
& 0 \leqslant \rho_{e} \leqslant 1 & e=1,2, \ldots, N_{e}
\end{array}
$$

where $\rho \in \mathbb{R}^{N_{e}}$ are the design variables of the optimization problem, $V(\overline{\boldsymbol{\rho}})$ is the structural volume, which depends on the physical relative densities $\bar{\rho} \in \mathbb{R}^{N_{e}}, N_{e}$ is the number of finite elements in the finite element mesh, $V_{e}$ is the structural volume of finite element $e, \sigma_{e q}^{(k)}(\bar{\rho})$ is the von Mises equivalent stress at point $k, \sigma_{y}$ is the yield stress, $N_{k}$ is the number of points where the von Mises equivalent stress is computed, $\mathbf{K}(\overline{\boldsymbol{\rho}})$ is the global stiffness matrix, $\mathbf{U}(\overline{\boldsymbol{\rho}})$ is the global displacement vector and $\mathbf{F}$ is the global load vector. The local stiffness matrix of element $e$ is computed with the Solid Isotropic Material with Penalization (SIMP) scheme [40], as $\mathbf{k}_{e}\left(\bar{\rho}_{e}\right)=\left(\rho_{\min }+\left(1-\rho_{\min }\right) \bar{\rho}_{e}^{p}\right) \mathbf{k}_{e}^{0}$, where $\rho_{\min }=1 \times 10^{-9}$ is adopted to avoid singularity of the global stiffness matrix when solving for equilibrium, $p$ is a penalization factor and $\mathbf{k}_{e}^{0}$ is the $e$-th local stiffness matrix of base material. In this paper, $p=3$ is employed, as also adopted by several other authors in stress-based design [41].

The von Mises equivalent stress at any point $k$ is computed based in [1], and can be written as

$$
\sigma_{e q}^{(k)}(\bar{\rho})=\sqrt{\sigma_{k}^{T}(\bar{\rho}) \mathbf{M} \sigma_{k}(\bar{\rho})}
$$

where $\sigma_{k}(\bar{\rho})$ is the stress vector at any point $k$, computed as

$$
\boldsymbol{\sigma}_{k}(\overline{\boldsymbol{\rho}})=f_{\sigma}\left(\bar{\rho}_{k}\right) \mathbf{C}^{0} \mathbf{B}_{k} \mathbf{u}_{k}(\overline{\boldsymbol{\rho}})
$$

and $\mathbf{M}$ is a constant matrix, defined, for plane problems, as

$$
\mathbf{M}=\left[\begin{array}{ccc}
1 & -\frac{1}{2} & 0 \\
-\frac{1}{2} & 1 & 0 \\
0 & 0 & 3
\end{array}\right]
$$


In Equation (3), $\mathbf{C}^{0}$ is the constitutive matrix of base material, $\mathbf{B}_{k}$ is the strain-displacement transformation matrix evaluated at point $k$ and $\mathbf{u}_{k}(\overline{\boldsymbol{\rho}})$ is the local displacement vector of the element which contains point $k$.

The stress interpolation function $f_{\sigma}\left(\bar{\rho}_{k}\right)$, necessary for stress computation, Equation (3), must be properly chosen to avoid the singularity phenomenon [8]. In this paper, the $\varepsilon$-relaxed approach [42] is employed, adapted to solve density-based problems by [2], with $f_{\sigma}\left(\bar{\rho}_{k}\right)=\frac{\bar{\rho}_{k}}{\varepsilon\left(1-\bar{\rho}_{k}\right)+\bar{\rho}_{k}}$. It is demonstrated that parameter $\varepsilon$ can be properly chosen, in order to improve accuracy of stress evaluation at topology boundaries; hence, this parameter is not fixed a priori. The choice of parameter $\varepsilon$ is addressed in subsection 3.1 .

\subsection{Robust formulation}

The robust formulation proposed in this work is based on the approach presented in [37], where three relative density fields are considered: dilated $\overline{\boldsymbol{\rho}}^{(d)}$, intermediate $\overline{\boldsymbol{\rho}}^{(i)}$ and eroded $\overline{\boldsymbol{\rho}}^{(e)}$. A topology optimization problem of volume minimization subjected to local stress constraints is idealized considering that von Mises stresses are satisfied at every point where stress is computed, in each of the three distinct relative density fields. This problem can be written, in discrete form, as

$$
\begin{array}{clc}
\underset{\rho}{\operatorname{Min} .} & V\left(\overline{\boldsymbol{\rho}}^{(d)}\right)=\sum_{e=1}^{N_{e}} V_{e} \bar{\rho}_{e}^{(d)} & \\
\text { s. t. } & \frac{\sigma_{e q}^{(k)}\left(\overline{\boldsymbol{\rho}}^{(e)}\right)}{\sigma_{y}}-1 \leqslant 0 & k=1,2, \ldots, N_{k} \\
& \frac{\sigma_{e q}^{(k)}\left(\overline{\boldsymbol{\rho}}^{(i)}\right)}{\sigma_{y}}-1 \leqslant 0 & k=1,2, \ldots, N_{k} \\
& \frac{\sigma_{e q}^{(k)}\left(\overline{\boldsymbol{\rho}}^{(d)}\right)}{\sigma_{y}}-1 \leqslant 0 & \\
& \mathbf{K}\left(\overline{\boldsymbol{\rho}}^{(e)}\right) \mathbf{U}\left(\overline{\boldsymbol{\rho}}^{(e)}\right)=\mathbf{F} & \\
& \mathbf{K}\left(\overline{\boldsymbol{\rho}}^{(i)}\right) \mathbf{U}\left(\overline{\boldsymbol{\rho}}^{(i)}\right)=\mathbf{F} & \\
& \mathbf{K}\left(\overline{\boldsymbol{\rho}}^{(d)}\right) \mathbf{U}\left(\overline{\boldsymbol{\rho}}^{(d)}\right)=\mathbf{F} & \\
& 0 \leqslant \rho_{e} \leqslant 1 & e=1,2, \ldots, N_{k}, \\
\end{array}
$$

where volume of the dilated structure, the largest among all volumes, is minimized ${ }^{1}$.

In such robust formulation, physical relative densities are given by three distinct fields: an eroded, an intermediate and a dilated. The main idea consists in reducing manufacturing sensitivity of intermediate structures by ensuring that eroded and dilated optimized structures satisfy local stress constraints as well. As uniform fabrication errors are unavoidable, like under-etching or over-etching [37], the structure would maintain good performance, considering

\footnotetext{
${ }^{1}$ As discussed in [36] and [37], if one works directly with limiting/minimizing the structural volume of the intermediate structure, numerical instabilities may arise, leading to non-optimal solutions. Working with the dilated structure, instead, avoids such numerical instabilities and guarantees minimization of intermediate and eroded structural volumes as well.
} 
variations that respect limitations imposed by eroded and dilated designs, since these designs satisfy local failure criterion as well.

It is demonstrated, however, that even though eroded, intermediate and dilated structures locally satisfy stress constraints, the stress feasibility for designs between eroded and intermediate and between intermediate and dilated structures can only be ensured with reasonable choice of model parameters. When a fixed grid approach is employed for topology optimization (as in this work), artificial stress concentrations at jagged interfaces arise, as emphasized in [17] and later in section 3, leading to stress inaccuracy and large stress oscillations for uniform boundary variations and, hence, leading to non-robust solutions. One of the main objectives of this work is to demonstrate that stress accuracy and low stress oscillations for uniform boundary variations, as well as robust intermediate designs, can be achieved under reasonable assumptions regarding stiffness and stress interpolation functions, as well as control of width of soft transition boundaries, as discussed later in section 3.

The main difference to the deterministic formulation is solution of two additional equilibrium equations per step of the optimization problem, as discussed in [37], and in the consideration of (three times) larger number of local stress constraints. If there is, for instance, one point of stress computation per finite element in the finite element mesh, and the whole set of local stress constraints is considered, we have three local stress constraints per finite element. Considering a finite element mesh with 10k elements, for instance, we would have an optimization problem with 10k design variables and 30k stress constraints.

\subsection{Threshold projection}

Physical relative densities are computed by density filtering with threshold projection, as presented in [37]. The expression of the threshold projection, used to compute physical relative density $\bar{\rho}_{e}$, is given by

$$
\bar{\rho}_{e}=\frac{\tanh (\beta \eta)+\tanh \left(\beta\left(\tilde{\rho}_{e}-\eta\right)\right)}{\tanh (\beta \eta)+\tanh (\beta(1-\eta))},
$$

where $\tilde{\rho}_{e}$ is the filtered relative density of element $e$, obtained from a linear projection

$$
\tilde{\rho}_{e}=\frac{\sum_{i \in \vartheta_{e}} w\left(\mathbf{x}_{i}\right) V_{i} \rho_{i}}{\sum_{i \in \vartheta_{e}} w\left(\mathbf{x}_{i}\right) V_{i}}
$$

over the design variables $\rho$, in a circular neighborhood $\vartheta_{e}$, centred in element $e$, which contains all the elements whose center is within a radius $R$ specified by the designer. 
In this paper, the linear weighting function is employed; it is defined as

$$
w\left(\mathbf{x}_{i}\right)=R-\left\|\mathbf{x}_{i}-\mathbf{x}_{e}\right\|
$$

where $\mathbf{x}_{i}$ are the coordinates of the center of element $i$ and $\mathbf{x}_{e}$ are the coordinates of the center of the neighborhood $\vartheta_{e}$.

The parameter $\beta>0$, in Equation (6), controls the nonlinearity of $\bar{\rho}_{e}$ with respect to $\tilde{\rho}_{e}$. For $\beta \rightarrow 0$ we have a linear behavior between physical and filtered relative densities, whereas for $\beta \rightarrow \infty$ we have a Heaviside step function [37]. Later in section 3, we demonstrate the parameter $\beta$ plays an important role on accuracy of stress evaluation at jagged edges. We demonstrate that proper choice of $\beta$ can be made to ensure stress accuracy and low stress oscillation after uniform boundary variation.

The parameter $\eta \in[0,1]$, in Equation (6), defines the inflexion point of the projection, defining the amount of filtered relative densities $\tilde{\rho}_{e}$ projected to either 0 or 1 , for $\beta \rightarrow \infty$. For $\eta=0$, for instance, we recover the Heaviside step approximation proposed in [43], which ensures minimum length scale on the solid phase [37]. For $\eta=1$, we recover the modified Heaviside step approximation proposed in [40], which ensures minimum length scale on the void phase [37].

\subsection{Solution algorithm}

Regardless of which problem is addressed: deterministic or robust; it is well known that stress-constrained continuum topology optimization problems are very challenging to solve, due to the local nature of stress failure criteria, and its large nonlinearity with respect to design variables. Ideally, stress constraints should be satisfied at every point of the design domain, leading to an infinite number of constraints. Considering a finite element discretization and one point of stress evaluation per element, the number of stress constraints is finite, but extremely large (proportional to the number of design variables). Due to this fact, it is acknowledged, in the literature, that solutions of continuum stress-constrained problems are non-trivial and still a challenge, being subject of intensive research.

In a comprehensive literature review, we verified that the most common approaches to solve continuum stressconstrained problems are classified within three categories, depending on how the stress constraints are handled: 1) global approaches; 2) regional approaches; or 3) local approaches. Global and regional approaches are often formulated based on some aggregation function, like the P-norm function [2] or the Kreisselmeier-Steinhauser function [7]; whereas local approaches are often formulated based on some active set strategy [1] or some alternative technique, like the augmented Lagrangian method [4].

It is acknowledged that each approach has advantages and disadvantages; however, this work does not aim at comparing them. The authors want to emphasize that the robust problem, proposed in Equation (5), can be solved by 
any of these approaches, since the only difference between deterministic and robust problems, from a computational point of view, is in the number of stress constraints, which is three times larger in the robust case.

In this paper, we choose to handle the whole set of local stress constraints by means of the augmented Lagrangian method [44], without employing aggregation techniques. The augmented Lagrangian method has been employed, in the literature, to solve topology optimization problems subjected to local stress constraints based on density [3-5, 2427] and level set $[15,16]$ approaches.

The essential idea associated with the augmented Lagrangian method consists in transforming the original constrained optimization problem into a sequence of unconstrained (or less constrained) subproblems. The objective function of the optimization subproblems is the augmented Lagrangian function, which consists in the original objective function weighted by design constraints, each associated with one penalization parameter and one Lagrange multiplier. The augmented Lagrangian method, then, consists in a sequential approach: after solving the current subproblem, penalization parameters and Lagrange multipliers are updated; then, solution of the current subproblem and updated parameters are used as initial estimate for solving the next subproblem, and so on, until convergence; meaning that solution of the original optimization problem is achieved.

In this paper, the augmented Lagrangian function is defined considering all stress constraints, associated with each relative density field, such that:

$$
\begin{gathered}
L\left(\overline{\boldsymbol{\rho}}^{(e)}, \overline{\boldsymbol{\rho}}^{(i)}, \overline{\boldsymbol{\rho}}^{(d)}, \boldsymbol{\mu}, r\right)=\frac{N_{e}}{\sum_{e=1}^{N_{e}} V_{e}} V\left(\overline{\boldsymbol{\rho}}^{(d)}\right)+\frac{r}{2} \sum_{k=1}^{N_{k}}\left(\left\langle\frac{\mu_{k}^{(d)}}{r}+\frac{\sigma_{e q}^{(k)}\left(\overline{\boldsymbol{\rho}}^{(d)}\right)}{\sigma_{y}}-1\right\rangle^{2}\right. \\
\left.+\left\langle\frac{\mu_{k}^{(i)}}{r}+\frac{\sigma_{e q}^{(k)}\left(\overline{\boldsymbol{\rho}}^{(i)}\right)}{\sigma_{y}}-1\right\rangle^{2}+\left\langle\frac{\mu_{k}^{(e)}}{r}+\frac{\sigma_{e q}^{(k)}\left(\overline{\boldsymbol{\rho}}^{(e)}\right)}{\sigma_{y}}-1\right\rangle\right)^{2},
\end{gathered}
$$

where $\langle\cdot\rangle=\max (0, \cdot)$. The objective function is weighted by constant $\frac{N_{e}}{\sum_{e=1}^{N_{e}} V_{e}}$ for the purpose of normalization.

Each stress constraint is associated with a Lagrange multiplier $\mu_{k}$, where superscripts ${ }^{(d)},{ }^{(i)}$ and ${ }^{(e)}$ indicate which relative density field each Lagrange multiplier is associated with. In this paper, only one penalization parameter $r$ is considered for the whole set of local stress constraints.

Since bound constraints are not included in the augmented Lagrangian function, they must be explicitly considered 
in the optimization subproblems, defined as

$$
\begin{aligned}
& \underset{\boldsymbol{\rho}}{\operatorname{Min}} \quad L\left(\overline{\boldsymbol{\rho}}^{(e)}, \overline{\boldsymbol{\rho}}^{(i)}, \overline{\boldsymbol{\rho}}^{(d)}, \boldsymbol{\mu}^{(c)}, r^{(c)}\right) \\
& \text { s. t. } \quad \mathbf{K}\left(\overline{\boldsymbol{\rho}}^{(e)}\right) \mathbf{U}\left(\overline{\boldsymbol{\rho}}^{(e)}\right)=\mathbf{F} \\
& \mathbf{K}\left(\overline{\boldsymbol{\rho}}^{(i)}\right) \mathbf{U}\left(\overline{\boldsymbol{\rho}}^{(i)}\right)=\mathbf{F} \\
& \mathbf{K}\left(\overline{\boldsymbol{\rho}}^{(d)}\right) \mathbf{U}\left(\overline{\boldsymbol{\rho}}^{(d)}\right)=\mathbf{F} \\
& 0 \leqslant \rho_{e} \leqslant 1 \quad e=1,2, \ldots, N_{e}
\end{aligned}
$$

where the superscript ${ }^{(c)}$ indicates $c$-th optimization subproblem.

After solving $c$-th optimization subproblem, one can employ the solution $\left(\rho^{(c)}\right)^{*}$ of the current subproblem and current estimate of Lagrange multipliers $\boldsymbol{\mu}^{(c)}$ and penalization parameter $r^{(c)}$ to update the next estimate of Lagrange multipliers

$$
\begin{aligned}
& \left(\mu_{k}^{(e)}\right)^{(c+1)} \leftarrow\left\langle r^{(c)}\left(\frac{\sigma_{e q}^{(k)}\left(\left(\overline{\boldsymbol{\rho}}_{(c)}^{(e)}\right)^{*}\right)}{\sigma_{y}}-1\right)+\left(\mu_{k}^{(e)}\right)^{(c)}\right\rangle, \\
& \left(\mu_{k}^{(i)}\right)^{(c+1)} \leftarrow\left\langle r^{(c)}\left(\frac{\sigma_{e q}^{(k)}\left(\left(\overline{\boldsymbol{\rho}}_{(c)}^{(i)}\right)^{*}\right)}{\sigma_{y}}-1\right)+\left(\mu_{k}^{(i)}\right)^{(c)}\right\rangle, \\
& \left(\mu_{k}^{(d)}\right)^{(c+1)} \leftarrow\left\langle r^{(c)}\left(\frac{\sigma_{e q}^{(k)}\left(\left(\overline{\boldsymbol{\rho}}_{(c)}^{(d)}\right)^{*}\right)}{\sigma_{y}}-1\right)+\left(\mu_{k}^{(d)}\right)^{(c)}\right\rangle,
\end{aligned}
$$

and penalization parameter

$$
r^{(c+1)} \leftarrow \begin{cases}\min \left(\gamma r^{(c)}, r_{\max }\right) & \text { if } \delta \sigma_{\max }^{(c)}>\omega \delta \sigma_{\max }^{(c-1)} \\ r^{(c)} & \text { otherwise }\end{cases}
$$

where $\gamma>1$ and $\omega<1$ are update parameters, $r_{\max }$ is an upper value of penalization parameter and $\delta \sigma_{\max }=$ $\left(\frac{\sigma_{\max }}{\sigma_{y}}-1\right)$, where $\sigma_{\max }$ represents the maximum value among all computed von Mises equivalent stresses. The value of penalization parameter $r$ is increased by a factor of $\gamma$ only if the maximum value of stress constraints reduces less than a factor of $\omega$, i.e., if there is reasonable progress regarding feasibility of the optimized topology, the penalization parameter is not updated, in order to avoid unnecessary increase of optimization subproblems nonlinearity.

This procedure is performed until both convergence criteria are simultaneously reached: 1) when maximum change on design variables becomes smaller than $\left.t o l_{\text {out }} ; 2\right)$ when feasibility is guaranteed, such that $\frac{\sigma_{\max }}{\sigma_{y}}-1<t_{0}$.

The value of $\beta$, from the threshold function, Equation (6), is increased through a continuation approach: the 
problem is initially solved considering a small value of $\beta$; then, the value of $\beta$ is increased and next problem is solved, considering solution of current problem, current Lagrange multipliers and current penalization parameter are used as initial estimates, and so on, until an upper value $\beta_{\max }$ is reached.

It is worth mentioning that the augmented Lagrangian function is continuous and has continuous first-order derivatives [45]; however, it has discontinuous second-order derivatives at $\frac{\mu_{k}^{(j)}}{r}+\frac{\sigma_{e q}^{(k)}\left(\bar{\rho}^{(j)}\right)}{\sigma_{y}}-1=0$, for $j \in\{e, i, d\}$, since the employed maximum operator $\langle\cdot\rangle$ is non-differentiable at zero. Thus, the optimization subproblems can be solved with any first-order algorithm able to handle bound constraints. In this paper, the subproblems are solved with a modified steepest descent method, as described in [26]. Sensitivity analysis is shown in the appendix.

\section{The problem of stress evaluation at jagged boundaries}

One of the main concerns when addressing stress-based topology optimization problems through fixed grid approaches (as in this paper) is in stress accuracy at jagged boundaries [17]. As highly emphasized in [17], when the boundary of the structure is not aligned with the finite element mesh, as usually happens in fixed grid approaches for topology optimization, non-physical stress concentrations appear at the jagged edges of the structure. As presented in [17], the wrong stress computation at jagged boundaries can negatively affect optimization, since stresses computed at these jagged boundaries are usually different from the stresses of the underlying smooth boundary.

The problem of stress evaluation at jagged edges is not only a concern in the field of stress-based topology optimization. This phenomenon was reported by several papers related to different fields (see, e.g., references [46-50]). In the scope of stress-based topology optimization, the problem of stress evaluation at jagged edges was already demonstrated in [17], where the accuracy of stress computation was investigated for rotation of the structure within a given fixed mesh, for different mesh sizes. However, since we aim at achieving manufacturing tolerant optimized designs, investigation of the accuracy of stress computation for slight uniform boundary variations remains fundamental.

In this section, the problem of stress evaluation at jagged boundaries is demonstrated by employing a voxel-based finite element model to evaluate the stresses of the well-known problem of plate with circular hole [51]. The problem is discretized with a 0/1 voxel-based mesh, simulating a void/solid ideal topology: relative density is equal to zero when the centroid of the element is inside the hole; and equal to one otherwise. Figure 1 illustrates the analyzed problem and discretization procedure.

The voxel-based model is discretized with four-node bi-linear square elements and stresses are computed at the center of each element. Input data are: Young's modulus of 1, thickness of 1, Poisson's ratio of 0.0. The maximum stress component $\sigma_{11}$ is evaluated through the voxel-based model, as shown in Figure 2, for three different meshes. The radius of the circular hole $R_{h}$ is increased from 0.09 to 0.11 , in order to simulate a manufacturing tolerance. 

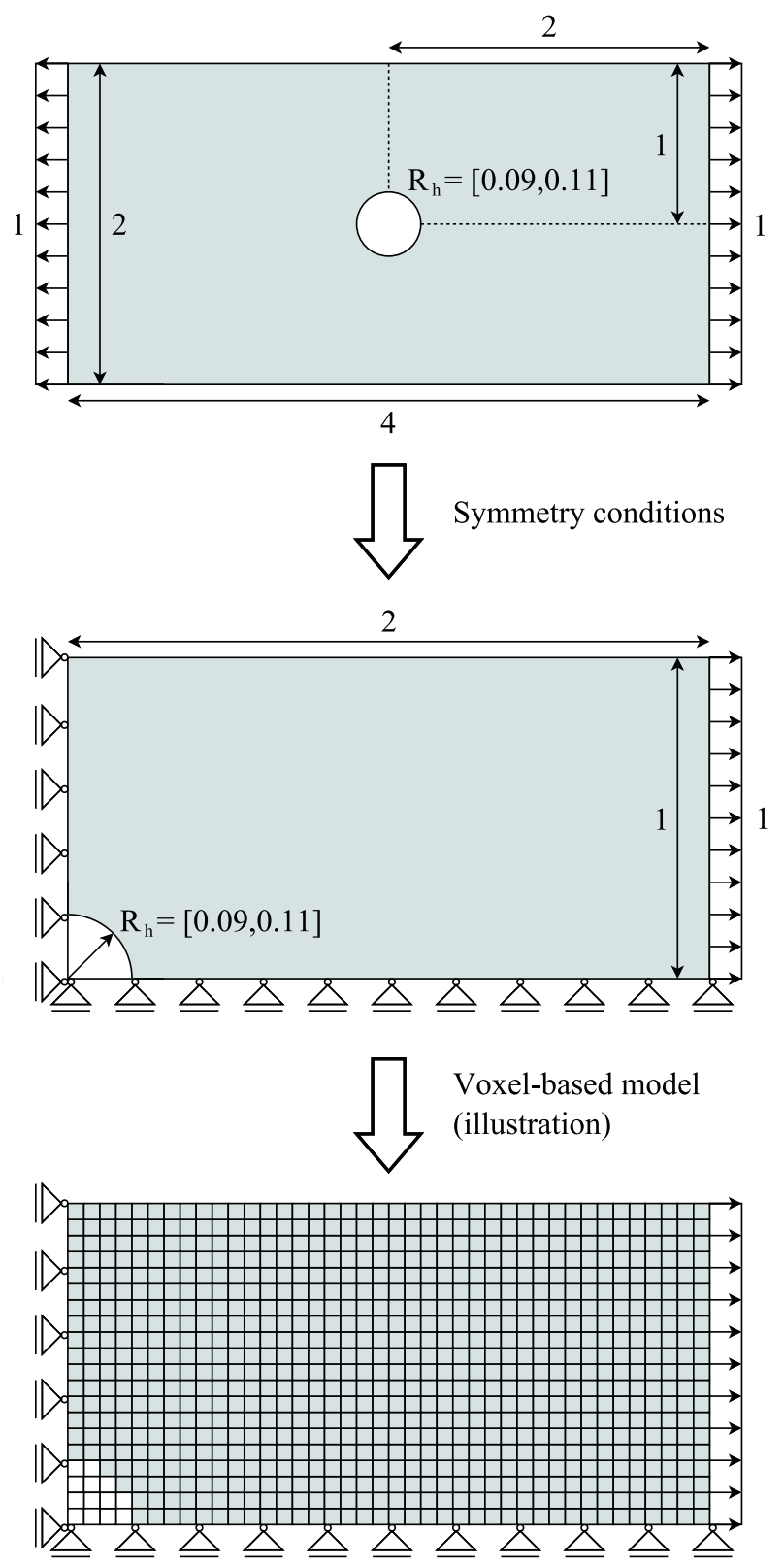

Figure 1: Problem of plate with circular hole, symmetry conditions and illustration of voxel-based model.

Figure 2 clearly demonstrates that the maximum value of $\sigma_{11}$ overestimates the reference solution with mesh refinement. Moreover, one can verify that the stress response strongly oscillates with increasing value of $R_{h}$ (i.e., with uniform boundary variation), contrary to what happens on the reference solution, that is absolutely smooth. Moreover, mesh refinements do not help at reducing these stress oscillations, rather the contrary.

In order to obtain manufacturing tolerant (robust) topologies, one has to improve the stress evaluation at jagged edges; i.e., one has to ensure both stress accuracy and low stress oscillation for uniform boundary variation. 


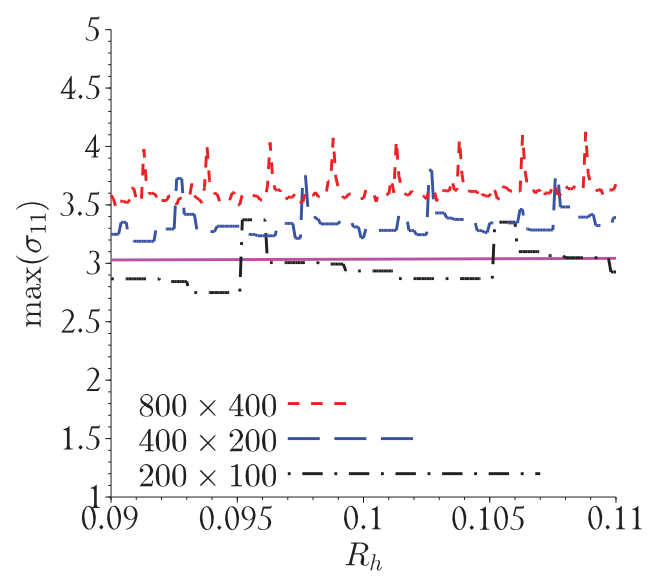

Figure 2: Maximum stress $\sigma_{11}$, computed for three different meshes and compared with the reference solution ( $\cong 3$, full line). Length of horizontal legend lines are representative of element size.

Some papers outside the field of topology optimization proposed techniques which may be applied to improve stress accuracy at jagged interfaces [46-50], most of them based on a post-processing step of stress smoothing. However, as discussed in [49], stress smoothing should be used with caution, since it becomes a compromise between mesh size and level of smoothing.

In the field of topology optimization, there is the Interior Value Extrapolation (IVE) technique, proposed by [17]. The IVE was developed to circumvent the problem of stress evaluation at jagged edges by extrapolating the stresses from the interior of the structure to its boundary. It is demonstrated, in [17], that the IVE approach works at better approximating the true stresses at the boundaries of the underlying structure when compared to the traditional stress evaluation approach.

In this paper, however, we choose to employ neither stress smoothing nor the IVE approach. Instead, an alternative two-step approach is proposed next, based on: 1) allowing a thin layer of intermediate material between solid and void phases; and 2) proper selection of parameter $\varepsilon$ for stress interpolation.

\subsection{Limiting sharpness of projected fields}

One can verify, in Figure 2, that the oscillatory behavior of the maxium stress has periodicity equal to the element size. In this subsection, the first step of a simple procedure for stress smoothing is presented, based on the consideration of a thin soft boundary with width equal to or slightly larger than the element size, i.e., the distance between two consecutive peaks of stress.

The procedure adopted herein to ensure a thin soft boundary between solid and void phases, consists of: 1) applying the linear density filter over the $0 / 1$ problem; 2) applying the threshold function while limiting sharpness of the projected field by limiting the value of $\beta$. Limiting the value of $\beta$ works by limiting incline of the threshold 
function, i.e., the larger the value of $\beta$, the smaller the layer of intermediate material at the boundaries of the structure. This subsection aims at demonstrating how the value of $\beta$ can be limited to ensure a soft boundary with width equal to at least the element size.

Let's consider 1D design domain with $x \in[0,2]$ and a design variable field with $\rho=0$ for $x<1$ and $\rho=1$ for $x \geqslant 1$, as shown in Figure 3 (a). Over the design variable field, one applies the linear density filter with filter radius $R=1$, obtaining the graph in Figure 3 (b) (see [37] for details).

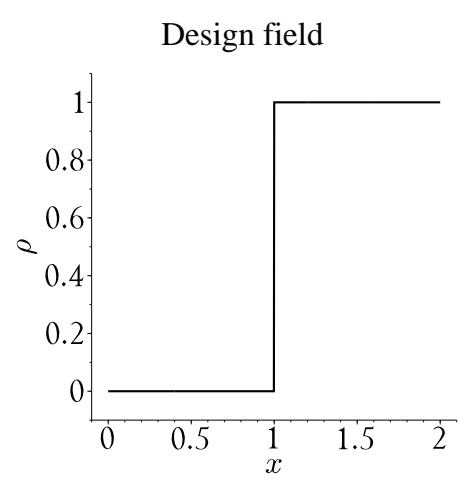

(a)

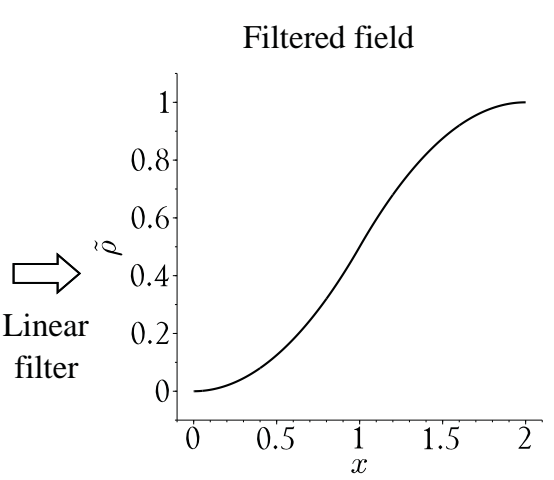

(b)

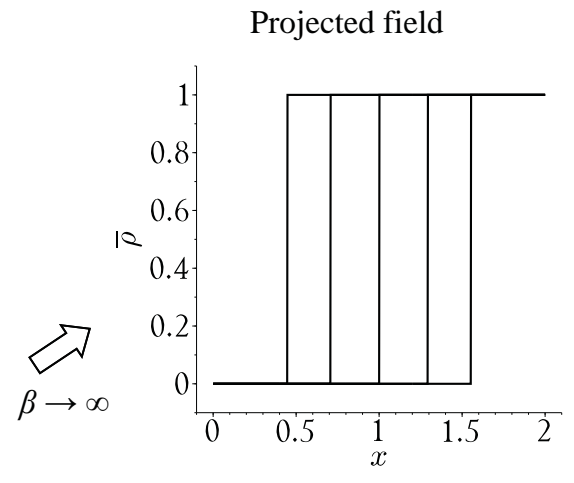

(c)

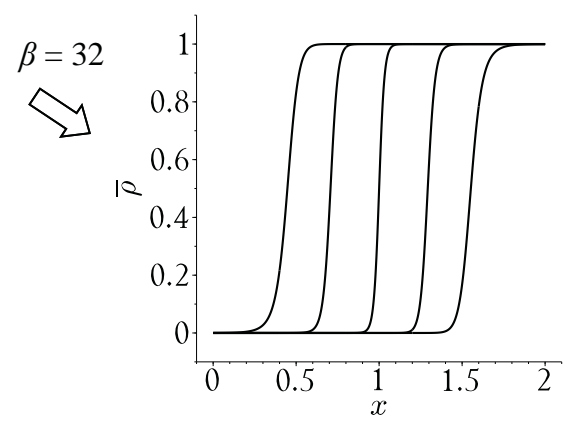

(d)

Figure 3: (a) design field; (b) filtered field and projected field for two different situations: (c) $\beta \rightarrow \infty$ and (d) $\beta=32$. The projected fields are evaluated at five distinct values of $\eta$, from left to right: $0.1,0.25,0.5,0.75,0.9$.

When applying the threshold function, Equation (6), for $\beta \rightarrow \infty$, sharp boundaries are obtained, as illustrated in Figure 3 (c). However, when a finite value of $\beta$ is employed, intermediate material between void and solid phases becomes evident, as illustrated in Figure 3 (d).

Analytical investigation demonstrates that the largest derivative of the threshold function with respect to $x$ always occurs for $\eta=0.5$ in $x=R$ (center of 1D design domain), illustrated in Figure 4 for filter radius $R=1$. Since this is the largest among all derivatives, this is the value that must be limited, in order to allow at least a soft transition boundary with width equal to the element size.

Through trivial algebra, one can demonstrate the incline of the threshold function over the filtered field, evaluated 


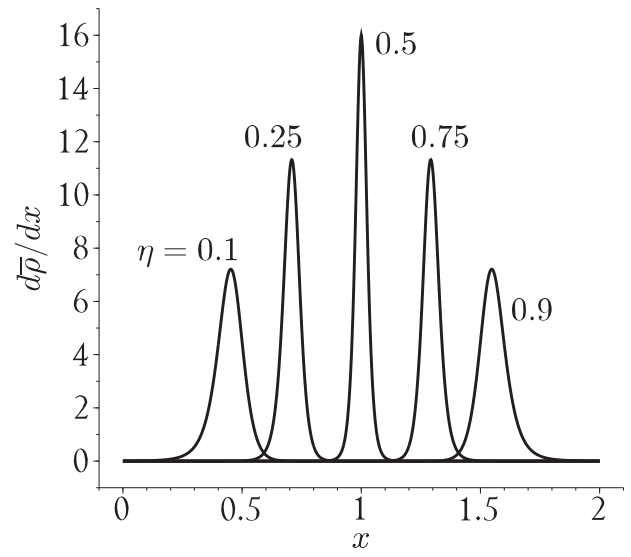

Figure 4: Derivatives of threshold function evaluated for $\beta=32$ (Figure 3 (d)) and five different values of $\eta$.

for $\eta=0.5$ and $x=R$, can be written as:

$$
\tan \left(\alpha_{\text {max }}\right)=\frac{0.5 \beta}{R \tanh (0.5 \beta)} .
$$

For large values of $\beta$, a simpler relation is found:

$$
\lim _{\beta \rightarrow \infty} \frac{\tan \left(\alpha_{\max }\right)}{\beta}=\frac{1}{2 R}
$$

whose asymptotic behavior is illustrated in Figure 5.

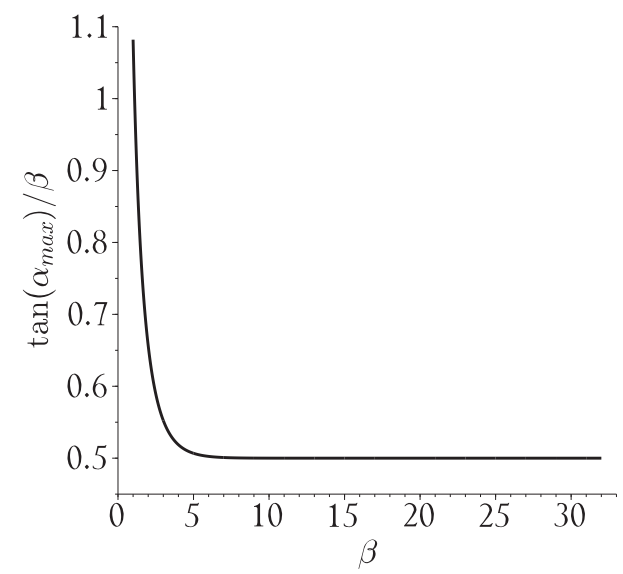

Figure 5: Illustration of asymptotic behavior of Equation (16), for $R=1$.

Based on Equation (16), the largest incline for a large value of $\beta$ can be defined as:

$$
\tan \left(\alpha_{\text {Max }}\right)=\frac{\beta}{2 R}
$$


Now, one can limit the projection considering the distance between two consecutive stress peaks (of one element only):

$$
\tan \left(\alpha_{l i m}\right)=\frac{1}{l_{e}} .
$$

Then, matching $\tan \left(\alpha_{\text {Max }}\right)=\tan \left(\alpha_{\text {lim }}\right)$, the $\beta_{\text {lim }}$ value is found as:

$$
\beta_{\text {lim }}=\frac{2 R}{l_{e}}
$$

The $\beta_{\text {lim }}$ value, defined in Equation (19), is employed to allow a transition boundary of intermediate material with the width corresponding to one element size. However, when sharpness of the threshold function is limited by employing a finite value of $\beta$, interpolation functions associated with stiffness and stress become very important, as demonstrated in next subsection. Hence, since the stiffness interpolation function was already defined beforehand (SIMP, with $p=3$ ), the choice of parameter $\varepsilon$, that governs the $\varepsilon$-relaxed function, should be properly chosen in order to ensure both stress accuracy and low stress oscillation.

\subsection{Choosing a proper stress interpolation parameter}

In this subsection, a numerical study is performed: the reference problem of the plate with circular hole is addressed again, considering now a thin layer of intermediate material between solid and void phases, i.e., the $\beta_{\text {lim }}$ scheme is employed.

A systematic procedure is adopted:

1. A $0 / 1$ design variable field is defined for hole radius $R_{h}=0.1$;

2. Linear density filtering is applied over the design variable field, considering $R=0.04$;

3. The threshold function is applied over the filtered field considering four values of $\beta\left(2 \times \beta_{\text {lim }}, \beta_{\text {lim }}, \beta_{\text {lim }} / 2\right.$ and $\left.\beta_{\text {lim }} / 4\right)$, in order to verify the stress behavior for different widths of soft transition boundary;

4. $\max \left(\sigma_{11}\right)$ is evaluated for every projected field considering the four values of $\beta$;

This procedure is performed for three sizes of finite element mesh: $200 \times 100,400 \times 200$ and $800 \times 400$; and five values of $\varepsilon: 0.05,0.1,0.2,0.3$ and 0.5 . The projected fields are obtained for $\eta \in[0.25,0.75]$, in order to achieve boundary variations with similar magnitude of our previous analyses, where $R_{h} \in[0.09,0.11]$. Results are shown in Figure 6.

Analyzing Figure 6, one can verify that choice of $\beta$ value and parameter $\varepsilon$ is not obvious. However, comparison with the reference solution demonstrates that these parameters can be properly chosen, in order to achieve consistent stress response at the jagged edges. 
$200 \times 100$
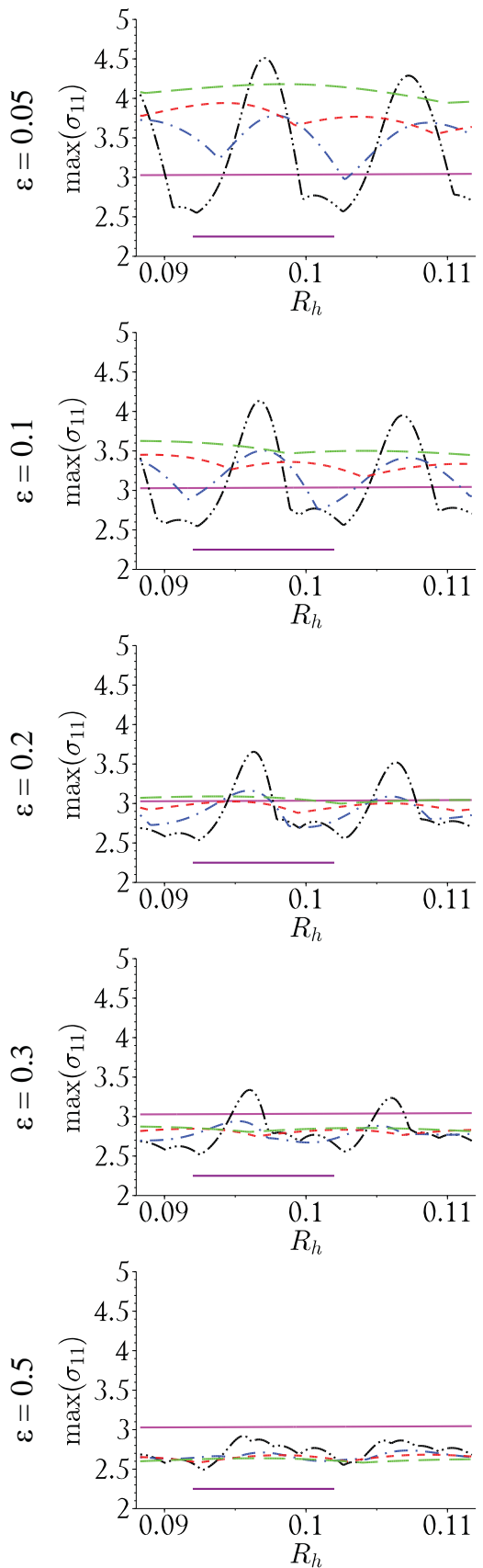

$400 \times 200$
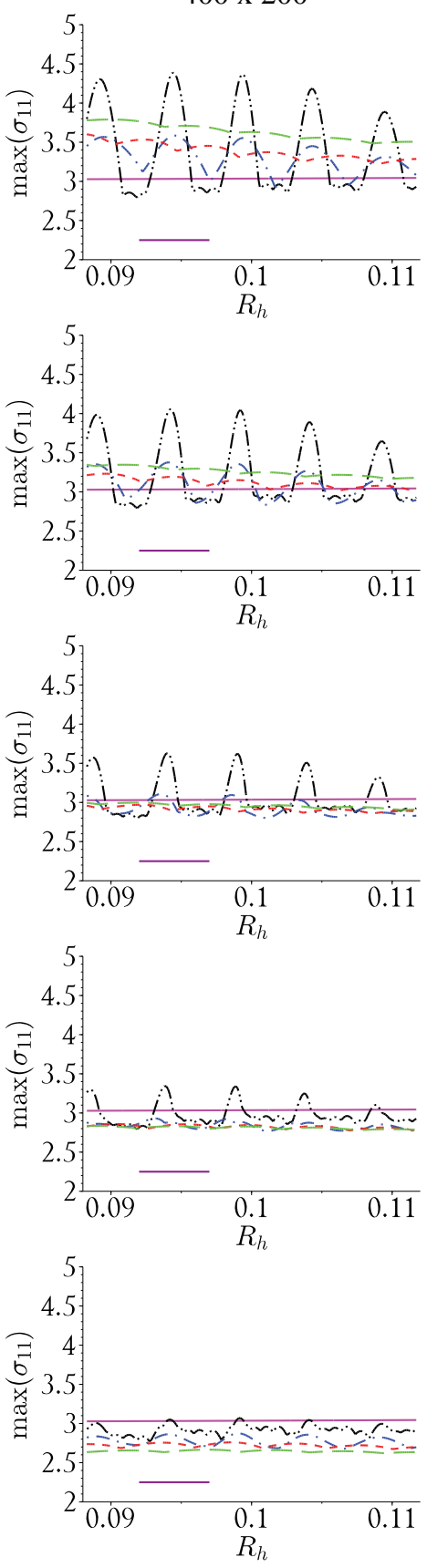

$800 \times 400$
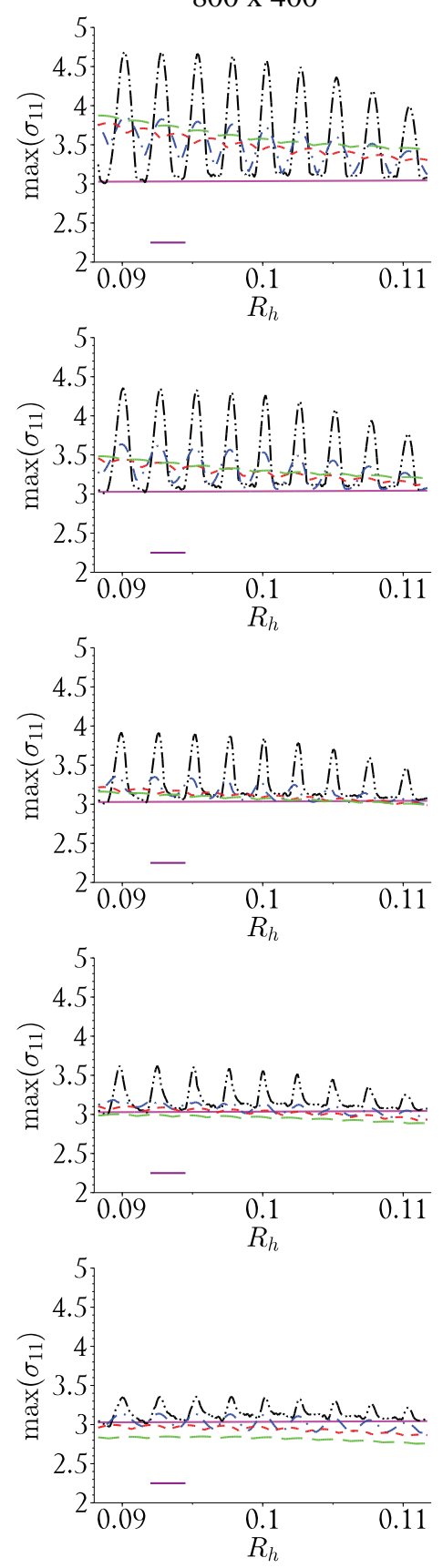

$$
\begin{aligned}
& \text { Reference } \\
& \text {-..- } \beta=2 \times \beta_{\text {lim }} \\
& -\cdot-\beta=\beta_{\text {lim }} \\
& ---\beta=\beta_{\text {lim }} / 2 \\
& --\beta=\beta_{\text {lim }} / 4
\end{aligned}
$$

Figure 6: Stress graphics for different values of $\varepsilon$ and mesh sizes. Each graphic is plotted for four different values of $\beta$ and compared with the smooth reference solution $(\cong 3)$. Length of horizontal legend lines are representative of element size. 
The numerical study demonstrates there is no need to ensure correct physics for the stresses at intermediate material (i.e., ensuring $\varepsilon \rightarrow 0$ ) if the goal is evaluation of stresses of the underlying solid/void structure.

The numerical study demonstrates that, by employing the traditional $\varepsilon$-relaxed function, adapted for continuum problems by [2], good agreement between stresses computed with the voxel-based mesh and stresses of the underlying reference problem can be ensured. However, proper choice of parameter $\varepsilon$ is necessary, as well as consideration of a proper layer of soft boundary of intermediate material.

It is worth mentioning that the traditional SIMP approach is employed in this study for stiffness interpolation, with standard parameter $p=3$. If different parameter for stiffness interpolation is employed, as well as a different stiffness interpolation function (e.g., the RAMP approach [52]), this numerical study would no longer be valid, and it would need to be redone, following the same procedure proposed in the beginning of this subsection. The same conclusion applies if a different stress interpolation function is employed, e.g., the qp-approach [6].

For the problems analyzed herein, one can verify that, in general, if the value of $\varepsilon$ is too small, the stresses at the jagged edges of the structure are overestimated. Moreover, small values of $\varepsilon$ cause strong stress oscillations after uniform boundary variation, which can negatively affect optimization convergence and quality of robust solution. On the other hand, if the value of $\varepsilon$ is too large, the stresses at the edges of the structure are underestimated. For this example, one verifies there is a proper choice of $\varepsilon$, in order to ensure both stress accuracy and low stress oscillation after uniform boundary variation, considering $\beta \leqslant \beta_{\text {lim }}$.

One can also verify that the larger the value of $\beta$, i.e., the smaller the amount of intermediate material between solid and void phases, the larger the stress oscillations due to uniform boundary variation. These oscillations are even more evident for small values of $\varepsilon$.

In the next section, stress-constrained robust optimization problems are addressed by employing $\varepsilon=0.2$. Based on graphics in Figure 6, this choice seems to provide a good compromise between stress accuracy and low stress oscillation due to uniform boundary variation, specially for $\beta \leqslant \beta_{\text {lim }} / 2$.

\section{Optimization results and post-processing: L-shaped problem}

In order to demonstrate applicability of proposed approach, the traditional L-shaped problem is solved. Hypotheses of plane stress are considered and four-node square elements are employed. Figure 7 illustrates geometry and boundary conditions.

Input data are: Young's modulus of $1 \times 10^{6}$, thickness of 0.001 , Poisson's ratio of 0.3 , yield stress of $\sigma_{y}=16 \times 10^{3}$, applied load of 0.3 , distributed over a boundary length of 0.05 to avoid stress concentrations, and filter's radius of $R=0.02$. A structured mesh with 102400 elements is considered (i.e., a $400 \times 400$ grid of finite elements). 


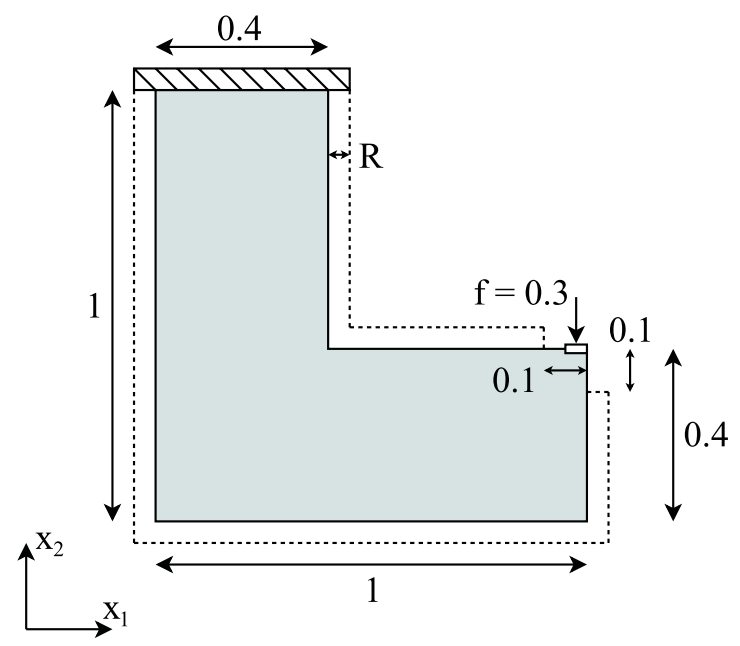

Figure 7: L-shaped design problem with geometrical dimensions and boundary conditions.

In order to limit sharpness of the projection, thus ensuring stress accuracy and avoiding excessive stress oscillations, a maximum value of $\beta_{\max }=\beta_{\lim } / 2$ is considered for the threshold function. For data adopted in this example, one can compute the $\beta_{\text {lim }}$ value as: $\beta_{\text {lim }}=2 \times R / l_{e}=2 \times 0.02 /(1 / 400)=16$, Equation (19). Thus, $\beta_{\max }=\beta_{\text {lim }} / 2=8$. Parameter $\beta$ is initially chosen as $\beta^{(1)}=1$ and updated through a three-step continuation approach, up to the $\beta_{\max }$ value, as: $\beta^{(1)}=1, \beta^{(2)}=4$ and $\beta^{(3)}=8$. The value of $\beta$ is updated when the convergence criteria are reached, as explained in the end of subsection 2.4 .

As illustrated in Figure 7, the design domain is extended by a width equal to the radius of the filter, $R$, except at support and load regions. This domain extension is filled with void elements, which are not considered in the analyses, only during application of the filter. This procedure, called filter boundary padding by [53], is adopted in order to alleviate possible boundary effects that may occur due to filtering.

Input data associated with optimization procedure: $r^{(1)}=0.003, r_{\max }=3000, \gamma=10$ and $\omega=0.8$, associated with the augmented Lagrangian method. $t_{\text {out }}=0.1$ and $t o l_{\sigma}=0.01$ as tolerances of outer problems. Subproblems are solved with algorithm presented in [26], with $n_{i t}^{\max }=20$ as the maximum number of iterations of a subproblem and $\operatorname{tol}_{\text {sub }}=0.01$ as tolerance of subproblems. Initial value of design variables: $\boldsymbol{\rho}^{(1)}=\mathbf{1}$.

In this example, the dilated, intermediate and eroded topologies are evaluated for $\eta_{d}=0.25, \eta_{i}=0.5$ and $\eta_{e}=0.75$, respectively. The optimized solution is obtained within 1222 iterations. Figure 8 shows optimized topologies, for the three density fields, with respective von Mises equivalent stress fields. Topologies are illustrated in gray scale images, where white represents zero density (void phase) and black represents unitary density (solid phase). Von Mises stresses are illustrated in color images, where blue represents zero stress and red represents maximum stress.

Analyzing Figure 8, one can verify that eroded, intermediate and dilated topologies are almost the same. The 

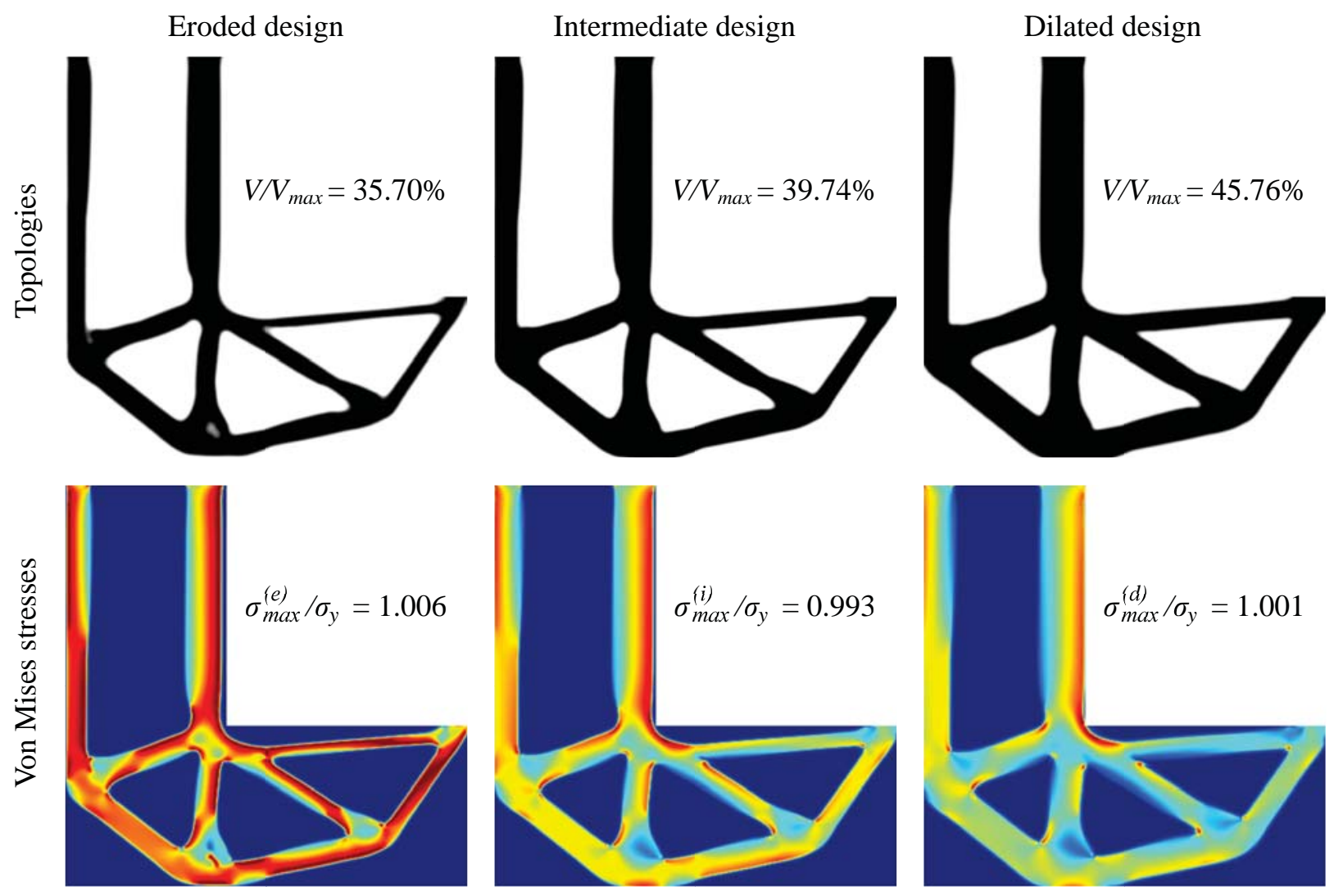

Figure 8: Topologies and respective von Mises equivalent stresses for eroded (left), intermediate (middle) and dilated (right) optimized structures.

only difference among the topologies is in the eroded design, where a small hole has appeared. Figure 8 also shows that the three structures satisfy local stress constraints in every point where von Mises equivalent stress is computed, considering the tolerance of $t o l_{\sigma}=1 \%$ with respect to yield stress. As expected, the eroded designs are very stressed systems, while the dilated designs have fewer (but still finite) points under maximum stress. It is also interesting to observe that there is a tendency to avoid the sharp corner present on the L-shaped design domain, in each of the three fields of relative densities. The influence of the small hole over the stress distribution of eroded design is addressed later during post-processing with body-fitted meshes.

Although eroded, intermediate and dilated structures clearly satisfy stress constraints, evaluation of the von Mises stresses for values of $\eta$ between $\eta_{d}$ and $\eta_{e}$ is necessary, in order to verify if the obtained intermediate structure is truly robust with respect to uniform boundary variations. Moreover, post-processing with body-fitted meshes is also recommendable, in order to verify if the stresses obtained through the voxel-based model (pixel-based topology) agree with the stresses obtained through the body-fitted model (smooth underlying topology).

Hence, two post-processing procedures are adopted and further compared: 1) Voxel-based post-processing scheme; 
and 2) Body fitted post-processing scheme.

The first and simplest post-processing procedure adopted herein is the voxel-based scheme. For the whole range of $\eta \in[0.0,0.8]$, the threshold function, for $\beta=8$ (achieved during optimization), is applied over the optimized filtered field, $\tilde{\boldsymbol{\rho}}^{*}$, and maximum von Mises equivalent stress is computed. This procedure provides a graphic $\eta \times \sigma_{\max } / \sigma_{y}$, which is further analyzed together with results from a body fitted post-processing scheme. Range of $\eta \in[0.0,0.8]$ is intentionally investigated, since it contains the limiting values of $\eta_{d}=0.25$ and $\eta_{e}=0.75$, employed during optimization. Values of $\eta$ larger than 0.8 are not employed during post-processing, because the structures becomes thinner and thinner as the value of $\eta$ is increased, leading to disconnected structures and extremely high stress values, completely hiding smaller stress contributions on the graphic.

The second post-processing procedure, the body-fitted scheme, is also performed within the interval $\eta \in[0.0,0.8]$. However, since a specific unstructured mesh is necessary for each different topology, a smaller number of values of $\eta$ is considered. This study is then performed for sixteen values of $\eta$, from $\eta=0.05$ to $\eta=0.8$, considering incremental steps of 0.05 .

The following procedure is adopted, in order to obtain smooth structures from the pixel-based topologies and further obtain unstructured body-fitted meshes:

1. A simple nodal averaging is performed over the optimized field of filtered densities, including the void element regions of the extended filtered domain;

2. For a given value of $\eta$, the smooth underlying topology is directly obtained from its contour plot extracted directly from the nodal averaged field of filtered densities;

3. A fine unstructured finite element mesh, comprised of six-node triangle elements, is generated over the smooth contour plot previously extracted, by employing the software Gmsh [54];

The procedure is illustrated in Figure 9.

After the mesh generation step, von Mises equivalent stresses are evaluated at the centroid of each triangular element (stresses at support region are not taken into account). The number of finite elements in each mesh varies. The average number of elements is approximately 78000 .

Figure 10 shows results obtained from both post-processing schemes.

Analyzing Figure 10, one can verify smooth stress behavior between $\eta_{d}=0.25$ and $\eta_{e}=0.75$, for both voxelbased and body-fitted approaches. Considering the voxel-based approach (solid line), one can observe the value of the normalized stresses are close to 1 within the whole interval between $\eta_{d}=0.25$ and $\eta_{e}=0.75$. It is also important to observe that, although different from each other, solutions from body-fitted meshes are close to solutions from voxelbased meshes, exceeding the stress constraint imposed during optimization up to $5 \%$ only. Hence, one can verify that 
Filtered field

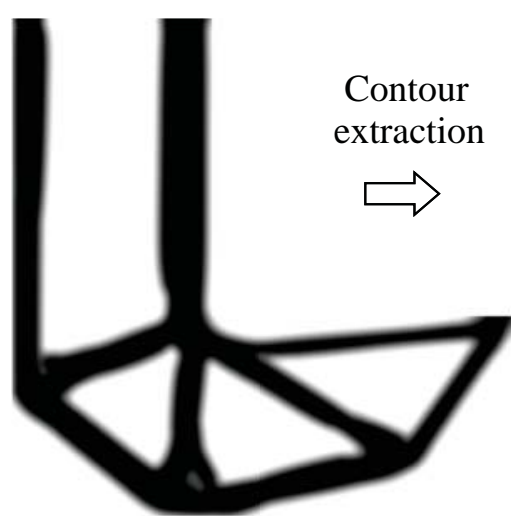

Contour plot for $\eta=0.5$

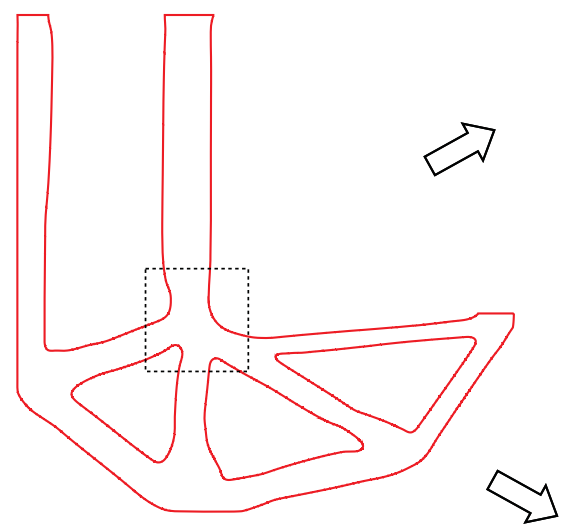

Contour plot over the intermediate topology

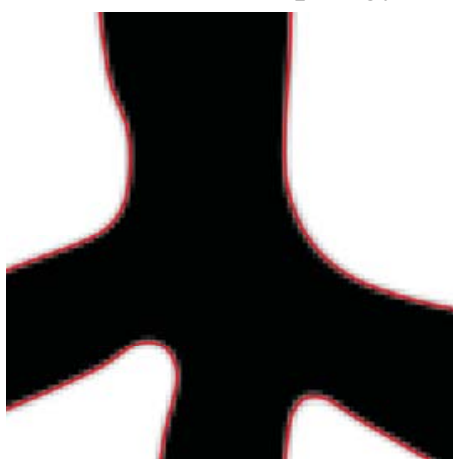

Body-fitted mesh

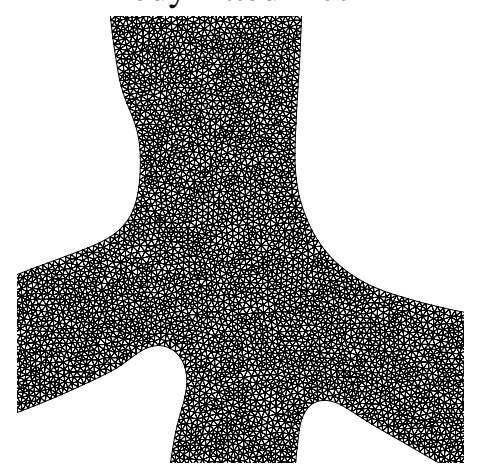

Figure 9: Adopted procedure for smooth contour extraction, for $\eta=0.5$.

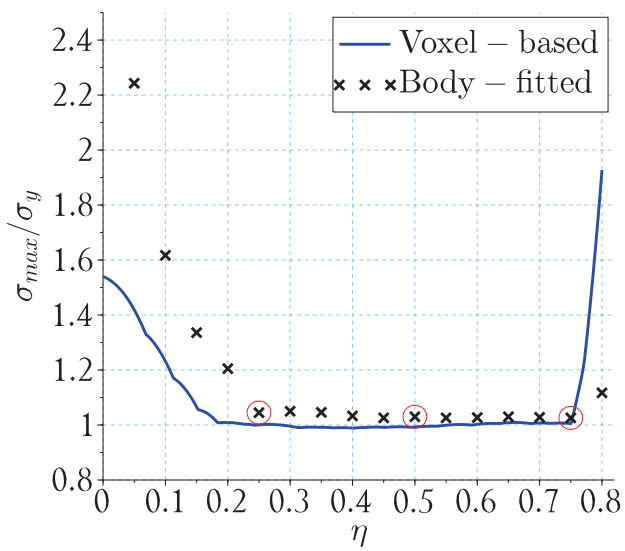

Figure 10: Post-processing von Mises equivalent stresses, for voxel-based and body-fitted schemes. Body-fitted results for eroded, intermediate and dilated designs are indicated by circles.

proposed approach is suitable for obtaining truly robust solutions, when appropriate values of $\beta_{\max }$ (threshold) and $\varepsilon$ (stress interpolation) are chosen.

For $\eta=0.75$ (eroded design), Figure 8 (upper left), a small hole arises, indicating a local difference between 
intermediate and eroded topologies. As expected, this small hole is also present in the contour plot associated with $\eta=0.75$, Figure 11 (a) and hence, in the body-fitted finite element model, Figure 11 (c). In order to verify the influence of this difference over the maximum von Mises stress, the same body-fitted post-processing scheme is performed disregarding the small hole, Figure 11 (b), where a maximum normalized von Mises stress of 1.026 is obtained, Figure 11 (d), i.e., there is no difference between maximum stresses when analyzing the eroded design with or without the small hole. Hence, in this example, this difference between eroded and intermediate topologies does not influence robustness of the intermediate design.

$$
\text { Contour plot for } \eta=0.75
$$



(a)

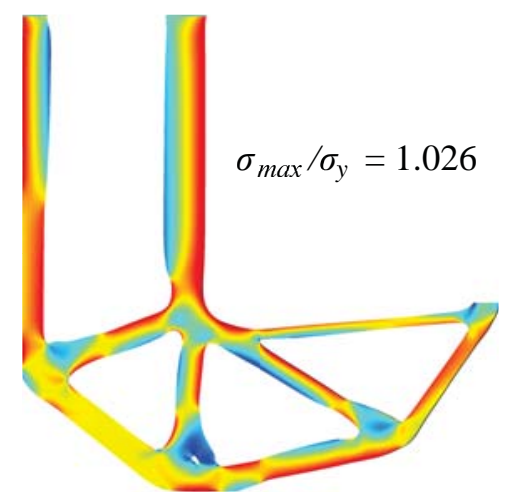

(c)

\section{Contour plot for $\eta=0.75$} disregarding hole



(b)



(d)

Figure 11: (a) contour plot for $\eta=0.75$; (b) contour plot for $\eta=0.75$ disregarding the small hole; (c) and (d) von Mises equivalent stress fields obtained with body-fitted models, from (a) and (b), respectively.

Outside the optimization range, i.e., for $\eta<0.25$ and $\eta>0.75$, different behaviors are observed. However, in these cases, the model is either too thick (leading to structures which hits the sharp corner) or too thin; hence, both cases are outside the sensible range and do not affect accuracy of optimization results.

Although nice agreement is observed in maximum von Mises stress behavior, for $\eta \in[0.25,0.75]$, it is also 
interesting to observe the similarity of the overall stress distributions. Figure 12 shows the von Mises equivalent stresses of voxel-based and body-fitted models, for $\eta=0.5$. Although presenting slightly different maximum von Mises stresses, the overall stress distributions are remarkably similar, due to successful employment of the $\beta_{\text {lim }}$ scheme associated with reasonable choice of stress interpolation parameter.

Note that the voxel-based von Mises stress model has a transition boundary between solid and void phases, i.e., there is a smooth stress transition from the finite stresses shown in the solid phase to the null stress of the void phase. This phenomenon, which is also observed in eroded and dilated structures, Figure 8, is a direct consequence of the employed thin layer of intermediate material. Although there is no physical meaning for these transition stresses, one can verify that they do not affect the overall quality of the stress distribution, once the stresses for voxel-based and body-fitted models are very similar, despite the fact that the body-fitted stress model has no such boundary effects, Figure 12. Moreover, the stress transition boundaries present in the voxel-based model can be properly reduced with mesh refinement, which would reduce the size of the element, and hence, increase the $\beta_{\text {lim }}$ value. A larger $\beta_{\text {lim }}$ value would allow use of larger $\beta_{\max }$ values for the threshold projection, without the undesired effect of stress oscillations due to uniform boundary variation; thus keeping a smooth stress response for even thinner layers of soft transition boundaries in voxel-based stress distributions.

It is also remarkable, although not unexpected in this example, that the optimization results in a structure that maintains constant maximum stress through even very large boundary variations; hence providing a truly robust design. It is worth noting that in this sense, the stress-based problem is very different from the compliance-based problem. As shown in [36], the eroded structure is always the most critical one in the compliance-based problem. On the other hand, we demonstrate that when addressing the stress-based problem, a remarkably different behavior is verified, where stress constraints are active within the whole range between eroded and dilated designs, indicating that all designs within the prescribed range are critical.

\subsection{Influence of mesh size}

In order to verify the influence of mesh size over the quality of robust solution, the L-shaped problem is solved with a coarser mesh, of 25600 four-node square elements (i.e., a $200 \times 200$ grid of finite elements). The problem is solved for $\beta_{\max }=8$, which coincides with the value of $\beta_{\text {lim }}$ in this case. Optimized solution is obtained within 911 iterations.

Figure 13 shows intermediate optimized topologies, for problems analyzed with coarse (25600) and fine (102400) meshes. Detail of the corner regions is also shown, where optimized filtered variables are placed together with the underlying smooth contour plots employed during post-processing. 


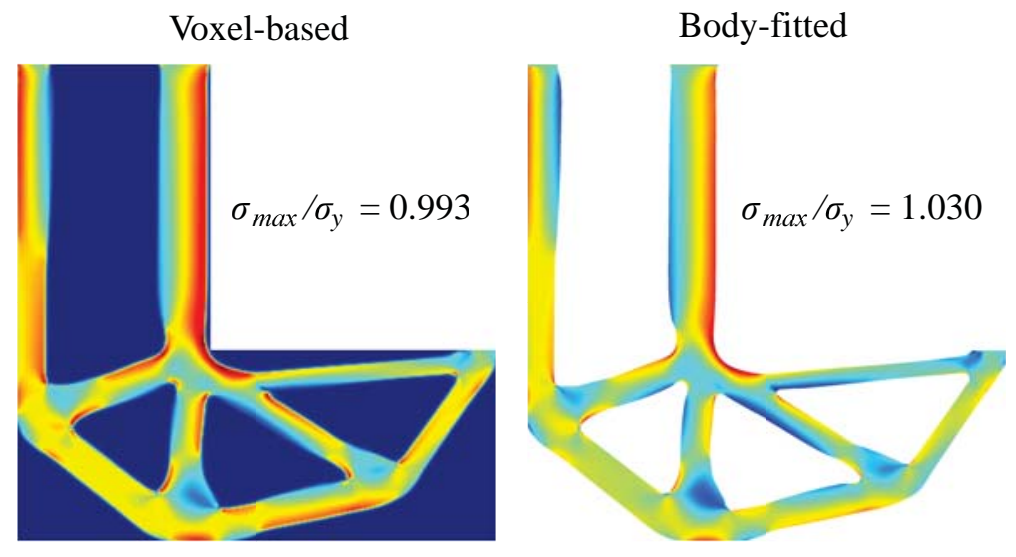

Figure 12: Von Mises equivalent stress fields obtained with voxel-based (left) and body-fitted (right) models, for $\eta=0.5$.


Figure 13: Robust intermediate designs (top) and zoom at the corner region of filtered fields (bottom), obtained for two different mesh sizes: $N_{e}=25600$ (left) and $N_{e}=102400$ (right). 
Although identical topologies are achieved after optimization, slightly different shapes are observed. Analyzing the contour plots, associated with underlying smooth topologies, one can verify two differences between solutions for coarse and fine meshes: 1) contour plots extracted from the finer filtered field are smoother; and 2) contour plot associated with $\eta=0.25$ (dilated design), slightly hits the sharp corner for the coarser result.

Despite these differences, the optimized design with coarser mesh also provides good post-processing results within the interval $\eta \in[0.25,0.75]$, as shown in Figure 14, except for the body-fitted model evaluated for $\eta=0.25$, which suffers from the effect of hitting the sharp corner.

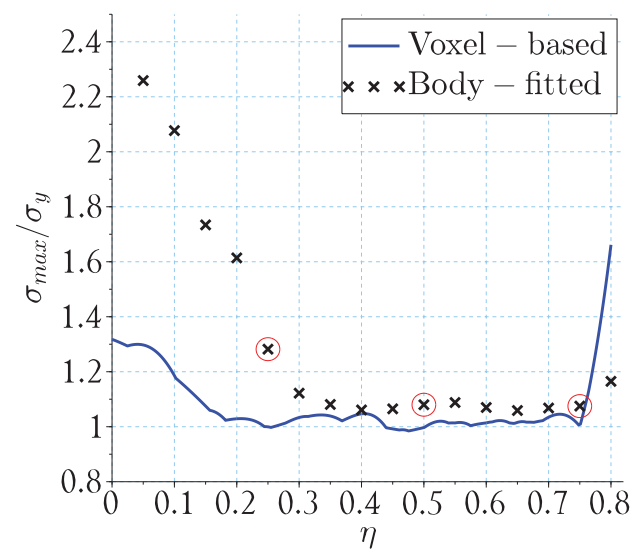

Figure 14: Post-processing von Mises equivalent stresses, for voxel-based and body-fitted schemes, for $N_{e}=25600$. Body-fitted results for eroded, intermediate and dilated designs are indicated by circles.

Von Mises equivalent stresses of the voxel-based model oscillate when the value of $\eta$ is increased, from 0.25 to 0.75. This oscillatory behavior is justified, since for the coarser mesh the $\beta_{\max }$ value, when applying the threshold function, coincides with $\beta_{\text {lim }}$. As shown in Figure 6 , for $\varepsilon=0.2$ and $\beta=\beta_{\text {lim }}$, small stress oscillations may still occur, independent of mesh size. Despite these oscillations, one can verify that the stress magnitude from voxelbased model does not largely exceed the limiting value of 1 , of the normalized stress constraints. Within the interval $\eta \in[0.25,0.75]$, stresses from the voxel-based model exceed the stress constraint imposed during optimization up to only $4.7 \%$.

When analyzing the stress responses obtained through the body-fitted post-processing scheme, larger differences are verified. As shown in Figure 13, on the bottom left, for $\eta=0.25$, the smooth structure, obtained directly from the $\eta=0.25$ contour plot, presents a small sharp corner. In this case, the stress responses obtained from both voxel-based and body-fitted models are questionable, since we cannot ensure mesh convergence in such a situation, due to the presence of a sharp corner in the physical model.

For $\eta>0.25$, the contour plots present much smoother behaviors at the corner region, allowing better comparisons 
between both models. Within the interval $\eta \in[0.3,0.75]$, stresses from the body-fitted model exceed the stress constraint up to $12.2 \%$.

By analyzing these results, one can verify that although good stress response can be obtained with a coarse mesh, a fine mesh is desirable to better describe the stress response of the underlying smooth structure. In Figure 13, identical topologies can be observed; however, slight differences in the shape of the underlying smooth structures seem to be crucial when aiming at achieving a good stress response. Moreover, a finer mesh seems to be better at describing the stress effects from the sharp corner, as one can see in Figure 13, where the contour plot for $\eta=0.25$ hits the corner for the coarser mesh, but is absolutely smooth for the finer mesh.

\section{Complementary numerical results}

For the sake of completeness, and as reference for future works in the area, two additional optimization problems are solved. Optimized results are post-processed with body-fitted meshes, by employing the same post-processing scheme presented earlier. Input data for the optimization algorithm and material properties are the same as considered in previous section, unless specified otherwise.

\subsection{Eye-bar problem}

The eye-bar problem [4, 9], Figure 15, is discretized with a distorted mesh of 52608 four-node bi-linear finite elements. The problem is solved by employing the symmetry conditions, following [4]. The vertical load is distributed over the upper boundary of the circular hole $\left(x_{2} \geqslant 0\right)$ according to function $f\left(x_{1}\right)=-x_{1}^{2}+1.5^{2}$, where $\left(x_{1}, x_{2}\right)=(0,0)$ is the center of the bar's eye.

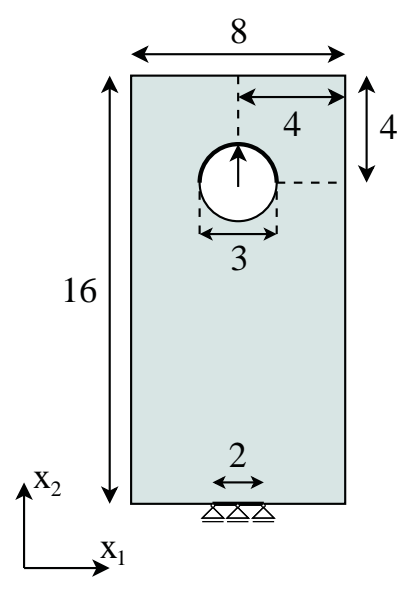

Symmetry conditions
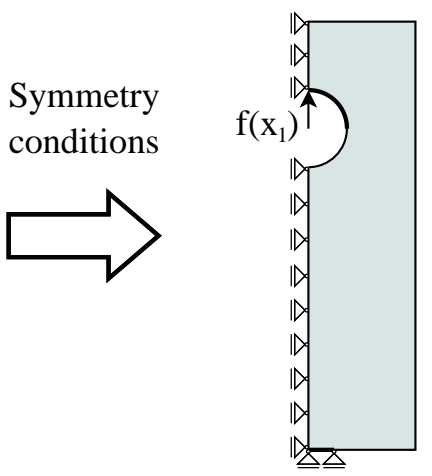

Figure 15: Eye-bar design problem with geometrical dimensions and boundary conditions. 
Input data are: filter's radius of $R=0.5$ and yield stress of $\sigma_{y}=5 \times 10^{3}$. The threshold values are $\eta_{d}=0.4$, $\eta_{i}=0.5$ and $\eta_{e}=0.6$. In this example, since a non-regular mesh is employed for discretizing the design domain, the $\beta_{\text {lim }}$ value is computed by considering the largest element of the mesh, as $\beta_{\text {lim }}=2 \times R / l_{e}=2 \times 0.5 / 0.065=15.4$. The $\beta$ value is updated through a three-step continuation approach, up to the $\beta_{\max }$ value, as: $\beta^{(1)}=1, \beta^{(2)}=4$ and $\beta^{(3)}=8$, with $\beta_{\text {max }} \cong \beta_{\text {lim }} / 2$. The optimized solution is obtained within 515 iterations.

Eroded, intermediate and dilated topologies with respective voxel-based von Mises stresses, as well as contour plots with respective body-fitted von Mises stresses, are shown in Figure 16. Figure 17 shows the graphs of postprocessing, where maximum von Mises equivalent stresses are computed for voxel-based and body-fitted meshes, within the range $\eta \in[0.4,0.6]$, i.e., the threshold range considered during optimization.

Analyzing Figure 16, one can observe that eroded, intermediate and dilated topologies are the same. Regarding the stress distributions, although the eroded structure is the most stressed one among the three designs, one can verify that intermediate and dilated structures show highly stressed regions as well, near the region where the distributed load ends.

Voxel-based and body-fitted von Mises stress distributions, Figure 16, show a low stressed region next to the boundary where the distributed load is applied. The von Mises stress goes to small values at this point, since the rim stress is going from compression to tension in this region. These results can be used to draw some parallels with [9], where the solution of the eye-bar stress-constrained problem shows some small holes at this region, indicating that the structural volume can be further minimized by removing the low stressed regions; however, at the cost of obtaining a highly imperfection sensitive design. The optimized results shown herein, in Figure 16, do not present the small holes at the load region, since the robust formulation is employed to solve the problem. As presented in [37], the robust formulation ensures a minimum length scale in both solid and void phases. In this example, appearing of the small holes is suppressed by use of a large filtering radius.

The post-processing stress graph, Figure 17, indicates that a truly robust solution is obtained, since all designs within the threshold range of $\eta \in[0.4,0.6]$ present a maximum von Mises equivalent stress that locally satisfies the stress constraints considering the prescribed tolerance of $1 \%$, for both voxel-based and body-fitted stress models. Moreover, the maximum von Mises equivalent stress shows smooth behavior for $\eta \in[0.4,0.6]$.

\subsection{Portal problem}

The portal problem [8, 23], Figure 18, is discretized with a grid-like mesh of 125538 four-node bi-linear square elements, corresponding to a $600 \times 300$ grid of finite elements, disregarding the elements whose coordinate of any node is inside the triangular cut, which are removed from the mesh. 

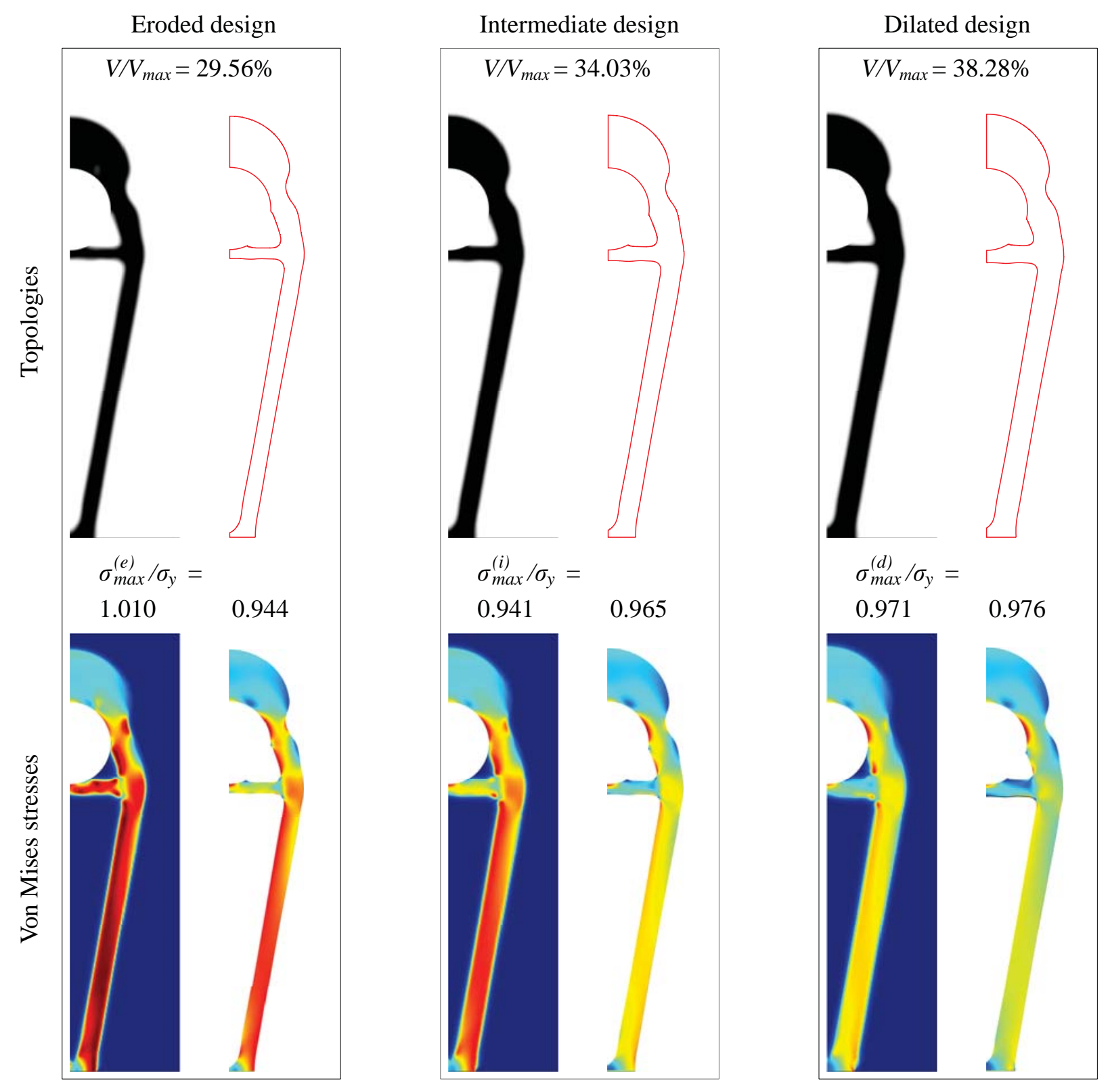

Figure 16: Eroded (left), intermediate (middle) and dilated (right) topologies with voxel-based von Mises stresses, and respective contour plots with body-fitted von Mises stresses, for the eye-bar problem.

Input data are: filter's radius of $R=0.016$, with filter boundary padding as indicated in Figure 18, and yield stress of $\sigma_{y}=16 \times 10^{3}$. The threshold values are $\eta_{d}=0.25, \eta_{i}=0.5$ and $\eta_{e}=0.75$. In this example, $\beta_{\text {lim }}=2 \times R / l_{e}=$ $2 \times 0.016 /(1.2 / 600)=16$. The $\beta$ value is updated through a three-step continuation approach, up to the $\beta_{\max }$ value, as: $\beta^{(1)}=1, \beta^{(2)}=4$ and $\beta^{(3)}=8$, with $\beta_{\max }=\beta_{\lim } / 2$. The applied load of 0.3 is distributed over a boundary length of 0.08 to avoid stress concentrations. The von Mises stresses next to the support regions are not considered during the optimization process nor the post-processing scheme. The optimized solution is obtained within 897 iterations. 


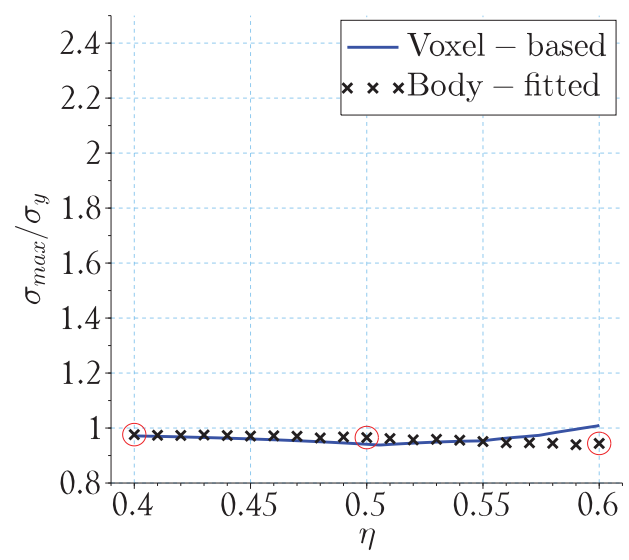

Figure 17: Post-processing von Mises equivalent stresses, for voxel-based and body-fitted schemes, for the eye-bar problem. Body-fitted results for eroded, intermediate and dilated designs are indicated by circles.

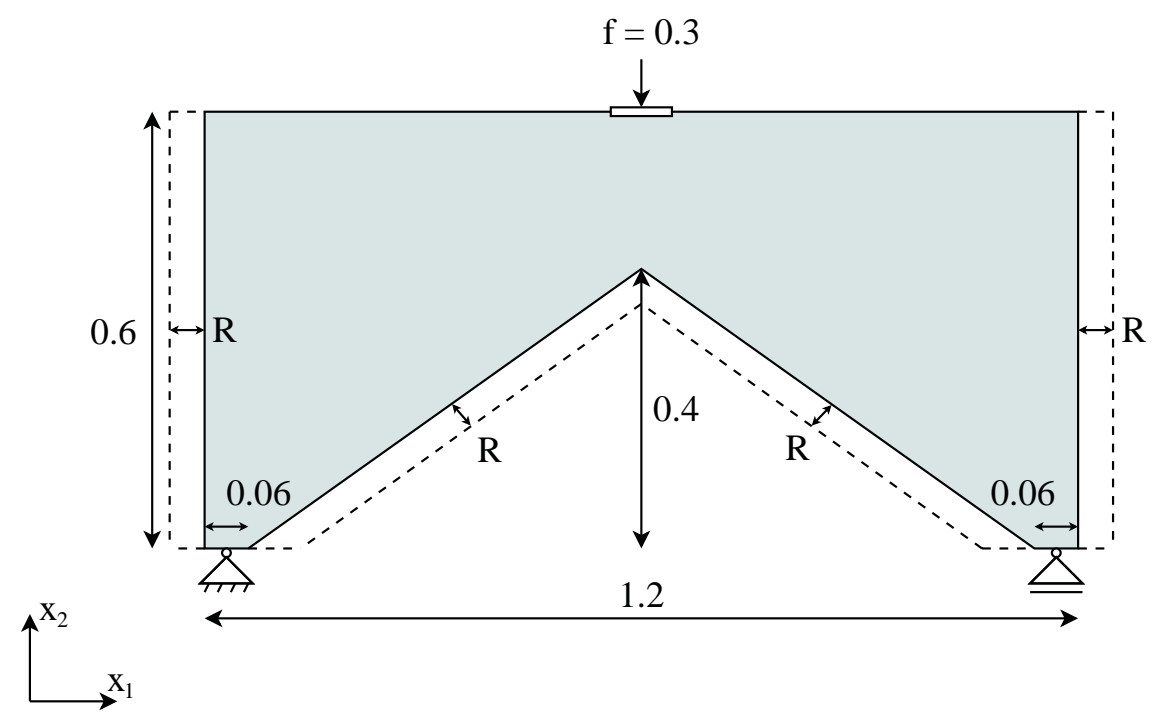

Figure 18: Portal design problem with geometrical dimensions and boundary conditions.

Figure 19 shows eroded, intermediate and dilated topologies, as well as respective contour plots and voxel-based and body-fitted von Mises equivalent stresses. Post-processing graphs of maximum von Mises equivalent stresses are shown in Figure 20, within the range $\eta \in[0.25,0.75]$.

Analyzing Figure 19, one can verify that eroded, intermediate and dilated topologies are the same. All structures properly avoided the re-entrant corner from the triangular cut, in the sense that the von Mises stresses at these regions locally satisfy the imposed stress constraints. Although the eroded structure is the most stressed one, both intermediate and dilated solutions present highly stressed regions, next to the re-entrant corner and next to the distributed applied load. Moreover, the body-fitted stress distributions are in agreement with the voxel-based stresses. 

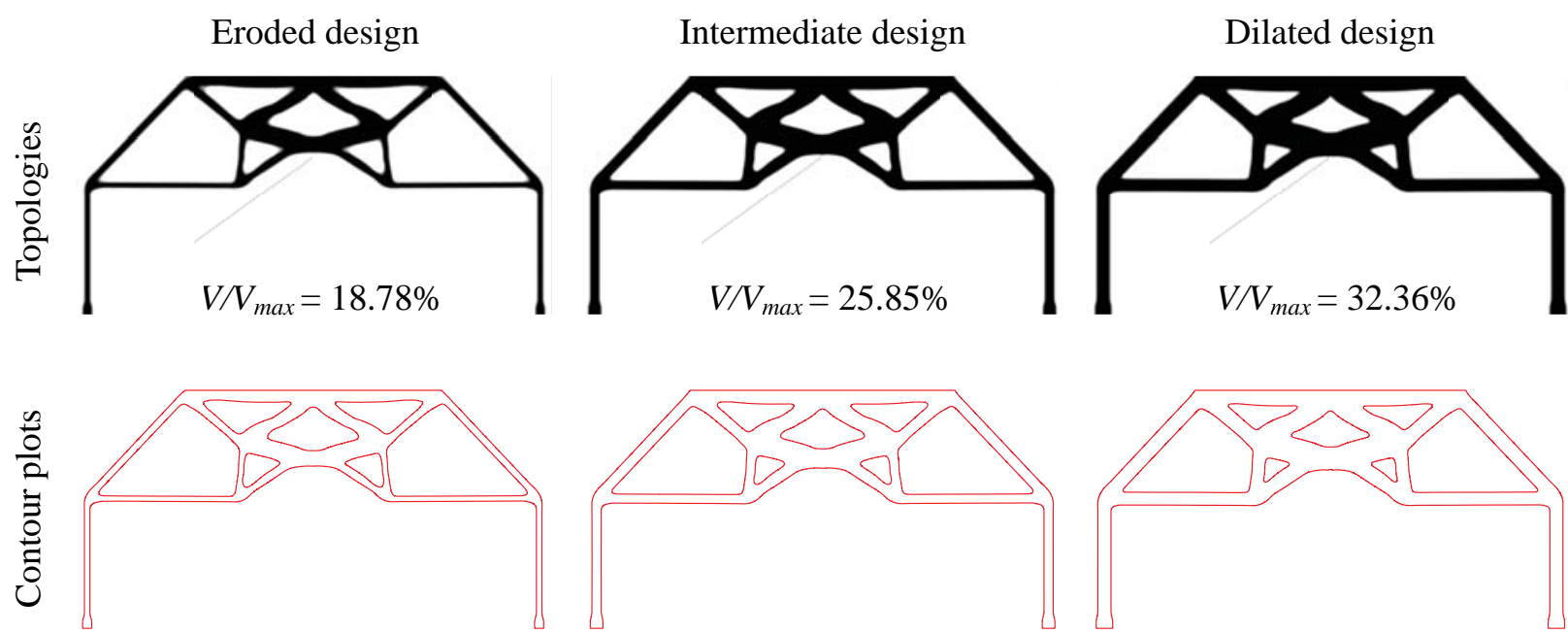

$\sigma_{\text {max }}^{(e)} / \sigma_{y}=1.010$

$\sigma_{\text {max }}^{(i)} / \sigma_{y}=0.979$

$\sigma_{\max }^{(d)} / \sigma_{y}=0.951$

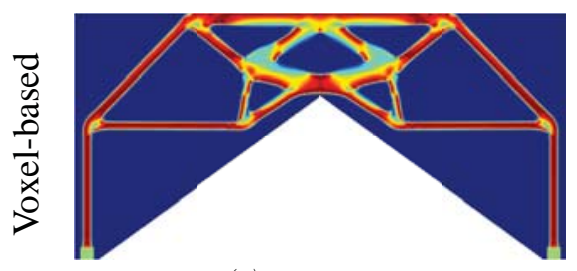

$\sigma_{\text {max }}^{(e)} / \sigma_{y}=1.048$
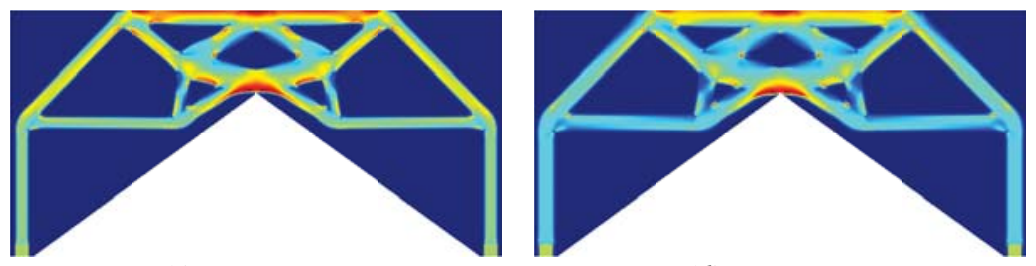

$\sigma_{\max }^{(i)} / \sigma_{y}=0.994$


$\sigma_{\max }^{(d)} / \sigma_{y}=1.046$

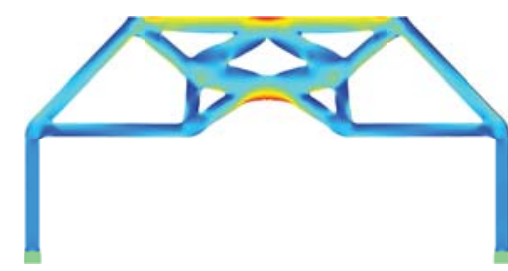

Figure 19: Eroded (left), intermediate (middle) and dilated (right) topologies (first line), respective contour plots (second line), voxel-based stresses (third line) and body-fitted stresses (fourth line), for the portal problem.

The post-processing stress graph, Figure 20, indicates smooth behavior for the maximum von Mises stress, within the range $\eta \in[0.25,0.75]$. The stresses from the voxel-based model exceed the stress constraint up to $1.3 \%$, whereas the stress constraint is exceeded up to $4.8 \%$ for the body-fitted model, indicating the obtained solution is indeed robust with respect to uniform boundary variations.

\section{Concluding remarks}

This work proposed and investigated a non-probabilistic robust formulation to tackle manufacturing uncertainties in stress-constrained topology optimization. The paper was divided in two closely related parts: 1) the problem of stress evaluation at jagged boundaries was addressed, and a simple and practical method for reducing stress oscillation and ensuring stress accuracy was proposed; 2) optimization problems were solved considering the proposed method, 


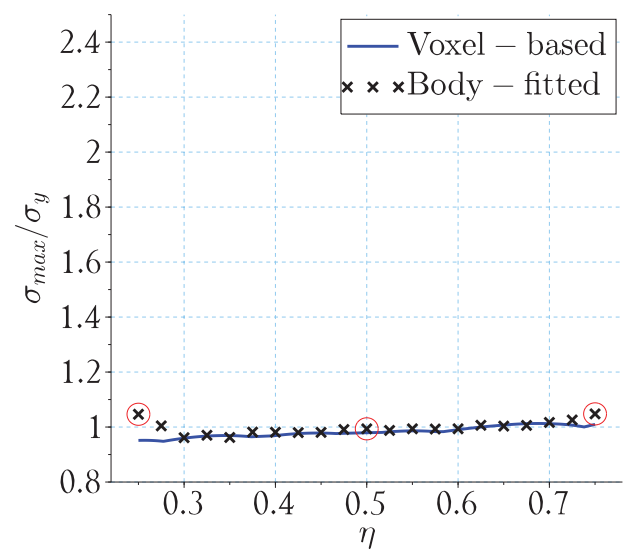

Figure 20: Post-processing von Mises equivalent stresses, for voxel-based and body-fitted schemes, for the portal problem. Body-fitted results for eroded, intermediate and dilated designs are indicated by circles.

and obtained results were properly validated.

The method proposed to alleviate jagged boundary effects consists in a consistent choice of stiffness interpolation, stress interpolation and sharpness of threshold projection. Under reasonable assumptions regarding these parameters, it was demonstrated that stress accuracy and low stress oscillation for uniform boundary variation can be ensured.

Several optimization problems were solved with the proposed robust approach, and the optimized structures were validated with proper post-processing based on body-fitted finite element models. It was demonstrated that the proposed robust approach is able to achieve manufacturing tolerant designs that satisfy stress constraints, regardless of the jagged interfaces.

A mesh dependency study was performed over the L-shaped design problem, demonstrating that identical topologies are obtained for two different mesh sizes, with slightly different shapes. However, the optimized solution for the finer mesh presented higher robustness with respect to manufacturing uncertainties, demonstrating that these slight differences in the shape of the optimized topology are crucial to ensure stress accuracy and, hence, robustness of the underlying smooth structure.

Resulting optimized structures are remarkable in the sense that their maximum stresses are insensitive to uniform boundary variations.

\section{Appendix: Sensitivity analysis}

This section develops sensitivity analysis, for evaluating the first-order derivative of the augmented Lagrangian function $L$ with respect to a design variable $\rho_{m}$, necessary for optimization with gradient-based algorithm.

The augmented Lagrangian function, Equation (9), can be properly rewritten in order to facilitate sensitivity 
analysis by the adjoint technique:

$$
L=V^{(d)}+\sum_{j \in P} L^{(j)}
$$

where $P=\{d, i, e\}$ is the set which contains dilated, intermediate and eroded fields of physical relative densities, $V^{(d)}$ is the normalized volume of the dilated structure, written as

$$
V^{(d)}=\frac{N_{e} \sum_{e=1}^{N_{e}} V_{e} \bar{\rho}_{e}^{(d)}}{\sum_{e=1}^{N_{e}} V_{e}},
$$

and $L^{(j)}$ is the term associated with the stress constraints, defined as

$$
L^{(j)}=\frac{r}{2} \sum_{k=1}^{N_{k}}\left\langle\frac{\mu_{k}^{(j)}}{r}+\frac{\sigma_{e q}^{(k)}\left(\overline{\boldsymbol{\rho}}^{(j)}\right)}{\sigma_{y}}-1\right\rangle^{2}+\lambda_{(j)}^{T}\left(\mathbf{K}\left(\overline{\boldsymbol{\rho}}^{(j)}\right) \mathbf{U}\left(\overline{\boldsymbol{\rho}}^{(j)}\right)-\mathbf{F}\right),
$$

where $\lambda_{(j)}$ are arbitrary vectors, since $\mathbf{K}\left(\overline{\boldsymbol{\rho}}^{(j)}\right) \mathbf{U}\left(\overline{\boldsymbol{\rho}}^{(j)}\right)-\mathbf{F}=\mathbf{0}$.

The derivative of the objective function (the augmented Lagrangian function) can be computed through a chain rule, as described in [40], in order to perform optimization in the non-physical design space:

$$
\frac{\partial L}{\partial \rho_{m}}=\sum_{n \in \vartheta_{m}} \frac{\partial V^{(d)}}{\partial \bar{\rho}_{n}^{(d)}} \frac{\partial \bar{\rho}_{n}^{(d)}}{\partial \tilde{\rho}_{n}} \frac{\partial \tilde{\rho}_{n}}{\partial \rho_{m}}+\sum_{j \in P} \sum_{n \in \vartheta_{m}} \frac{\partial L^{(j)}}{\partial \bar{\rho}_{n}^{(j)}} \frac{\partial \bar{\rho}_{n}^{(j)}}{\partial \tilde{\rho}_{n}} \frac{\partial \tilde{\rho}_{n}}{\partial \rho_{m}}
$$

where

$$
\frac{\partial V^{(d)}}{\partial \bar{\rho}_{n}^{(d)}}=\frac{N_{e} V_{n}}{\sum_{e=1}^{N_{e}} V_{e}}
$$

is the derivative of Equation (.2),

$$
\frac{\partial \bar{\rho}_{n}^{(j)}}{\partial \tilde{\rho}_{n}}=\frac{\beta\left(\operatorname{sech}\left(\beta\left(\tilde{\rho}_{n}-\eta_{j}\right)\right)\right)^{2}}{\tanh \left(\beta \eta_{j}\right)+\tanh \left(\beta\left(1-\eta_{j}\right)\right)}
$$

is the derivative of Equation (6), and

$$
\frac{\partial \tilde{\rho}_{n}}{\partial \rho_{m}}=\frac{w\left(\mathbf{x}_{m}\right) V_{m}}{\sum_{o \in \vartheta_{n}} w\left(\mathbf{x}_{o}\right) V_{o}}
$$

is the derivative of Equation (7).

The derivative of $L^{(j)}$, Equation (.3), with respect to a physical relative density $\bar{\rho}_{n}^{(j)}$, is directly computed as:

$$
\frac{\partial L^{(j)}}{\partial \bar{\rho}_{n}^{(j)}}=\sum_{k=1}^{N_{k}} h_{k}^{(j)} \frac{\partial \sigma_{e q}^{(k)}\left(\overline{\boldsymbol{\rho}}^{(j)}\right)}{\partial \bar{\rho}_{n}^{(j)}}+\lambda_{(j)}^{T}\left(\frac{\partial \mathbf{K}\left(\overline{\boldsymbol{\rho}}^{(j)}\right)}{\partial \bar{\rho}_{n}^{(j)}} \mathbf{U}\left(\overline{\boldsymbol{\rho}}^{(j)}\right)+\mathbf{K}\left(\overline{\boldsymbol{\rho}}^{(j)}\right) \frac{\partial \mathbf{U}\left(\overline{\boldsymbol{\rho}}^{(j)}\right)}{\partial \bar{\rho}_{n}^{(j)}}\right),
$$


where

$$
h_{k}^{(j)}=\left\langle\mu_{k}^{(j)}+r\left(\frac{\sigma_{e q}^{(k)}\left(\overline{\boldsymbol{\rho}}^{(j)}\right)}{\sigma_{y}}-1\right)\right\rangle \frac{1}{\sigma_{y}} .
$$

Through substituting Equation (3) in Equation (2), one can demonstrate that von Mises equivalent stress $\sigma_{e q}^{(k)}\left(\overline{\boldsymbol{\rho}}^{(j)}\right)$ is computed by multiplying the solid von Mises stress $\hat{\sigma}_{e q}^{(k)}\left(\overline{\boldsymbol{\rho}}^{(j)}\right)$ by the stress interpolation function $f_{\sigma}\left(\bar{\rho}_{k}^{(j)}\right)$, as follows:

$$
\sigma_{e q}^{(k)}\left(\overline{\boldsymbol{\rho}}^{(j)}\right)=f_{\sigma}\left(\bar{\rho}_{k}^{(j)}\right) \hat{\sigma}_{e q}^{(k)}\left(\overline{\boldsymbol{\rho}}^{(j)}\right)=f_{\sigma}\left(\bar{\rho}_{k}^{(j)}\right) \sqrt{\mathbf{u}_{k}^{T}\left(\overline{\boldsymbol{\rho}}^{(j)}\right) \mathbf{B}_{k}^{T} \mathbf{C}^{0} \mathbf{M} \mathbf{C}^{0} \mathbf{B}_{k} \mathbf{u}_{k}\left(\overline{\boldsymbol{\rho}}^{(j)}\right)}
$$

The derivative of the von Mises equivalent stress with respect to a physical relative density gives

$$
\frac{\partial \sigma_{e q}^{(k)}\left(\overline{\boldsymbol{\rho}}^{(j)}\right)}{\partial \bar{\rho}_{n}^{(j)}}=\frac{\partial f_{\sigma}\left(\bar{\rho}_{k}^{(j)}\right)}{\partial \bar{\rho}_{n}^{(j)}} \hat{\sigma}_{e q}^{(k)}\left(\overline{\boldsymbol{\rho}}^{(j)}\right)+\frac{f_{\sigma}\left(\bar{\rho}_{k}^{(j)}\right)}{\hat{\sigma}_{e q}^{(k)}\left(\overline{\boldsymbol{\rho}}^{(j)}\right)}\left(\mathbf{a}_{k}^{(j)}\right)^{T} \frac{\partial \mathbf{u}_{k}\left(\overline{\boldsymbol{\rho}}^{(j)}\right)}{\partial \bar{\rho}_{n}^{(j)}}
$$

where

$$
\left(\mathbf{a}_{k}^{(j)}\right)^{T}=\mathbf{u}_{k}^{T}\left(\overline{\boldsymbol{\rho}}^{(j)}\right) \mathbf{B}_{k}^{T} \mathbf{C}^{0} \mathbf{M} \mathbf{C}^{0} \mathbf{B}_{k}
$$

By substituting Equation (.11) in Equation (.8), relating the local displacement vector to the global displacement vector by employing the localization operator $\mathbf{H}_{k}$ [39], as $\mathbf{u}_{k}\left(\overline{\boldsymbol{\rho}}^{(j)}\right)=\mathbf{H}_{k} \mathbf{U}\left(\overline{\boldsymbol{\rho}}^{(j)}\right)$, and properly manipulating the resulting equation, one can write

$$
\begin{aligned}
\frac{\partial L^{(j)}}{\partial \bar{\rho}_{n}^{(j)}}= & \sum_{k=1}^{N_{k}} h_{k}^{(j)} \frac{\partial f_{\sigma}\left(\bar{\rho}_{k}^{(j)}\right)}{\partial \bar{\rho}_{n}^{(j)}} \hat{\sigma}_{e q}^{(k)}\left(\overline{\boldsymbol{\rho}}^{(j)}\right)+\lambda_{(j)}^{T} \frac{\partial \mathbf{K}\left(\overline{\boldsymbol{\rho}}^{(j)}\right)}{\partial \bar{\rho}_{n}^{(j)}} \mathbf{U}\left(\overline{\boldsymbol{\rho}}^{(j)}\right) \\
& +\left(\sum_{k=1}^{N_{k}} h_{k}^{(j)} \frac{f_{\sigma}\left(\bar{\rho}_{k}^{(j)}\right)}{\hat{\sigma}_{e q}^{(k)}\left(\overline{\boldsymbol{\rho}}^{(j)}\right)}\left(\mathbf{a}_{k}^{(j)}\right)^{T} \mathbf{H}_{k}+\lambda_{(j)}^{T} \mathbf{K}\left(\overline{\boldsymbol{\rho}}^{(j)}\right)\right) \frac{\partial \mathbf{U}\left(\overline{\boldsymbol{\rho}}^{(j)}\right)}{\partial \bar{\rho}_{n}^{(j)}} .
\end{aligned}
$$

In order to avoid the computation of the derivative of $\mathbf{U}\left(\overline{\boldsymbol{\rho}}^{(j)}\right)$ with respect to $\bar{\rho}_{n}^{(j)}$, the adoint vector, $\lambda_{(j)}$, is computed as

$$
\mathbf{K}\left(\overline{\boldsymbol{\rho}}^{(j)}\right) \lambda_{(j)}=-\sum_{k=1}^{N_{k}} h_{k}^{(j)} \frac{f_{\sigma}\left(\bar{\rho}_{k}^{(j)}\right)}{\hat{\sigma}_{e q}^{(k)}\left(\overline{\boldsymbol{\rho}}^{(j)}\right)} \mathbf{H}_{k}^{T} \mathbf{a}_{k}^{(j)}
$$

After computing the adjoint vector $\lambda_{(j)}$, the derivative of $L^{(j)}$ is computed as

$$
\frac{\partial L^{(j)}}{\partial \bar{\rho}_{n}^{(j)}}=h_{n}^{(j)} \frac{\partial f_{\sigma}\left(\bar{\rho}_{n}^{(j)}\right)}{\partial \bar{\rho}_{n}^{(j)}} \hat{\sigma}_{e q}^{(n)}\left(\overline{\boldsymbol{\rho}}^{(j)}\right)+\lambda_{(j, n)}^{T} \frac{\partial \mathbf{k}_{n}\left(\overline{\boldsymbol{\rho}}^{(j)}\right)}{\partial \bar{\rho}_{n}^{(j)}} \mathbf{u}_{n}\left(\overline{\boldsymbol{\rho}}^{(j)}\right)
$$

since the derivative of the stress interpolation function $f_{\sigma}\left(\bar{\rho}_{k}^{(j)}\right)$ is only different from zero for $\bar{\rho}_{k}^{(j)}=\bar{\rho}_{n}^{(j)}$, and the term associated with the derivative of the global stiffness matrix $\mathbf{K}\left(\overline{\boldsymbol{\rho}}^{(j)}\right)$ can be evaluated through local computations. In 
Equation (.15), $\lambda_{(j, n)}=\mathbf{H}_{k} \lambda_{(j)}$.

After computing the derivatives in Equations (.5), (.6), (.7) and (.15), the derivative of the augmented Lagrangian function $L$ with respect to a design variable $\rho_{m}$ is obtained through the chain rule shown in Equation (.4).

It is worth mentioning that three adjoint problems, Equation (.14), are solved to evaluate Equation (.15) for $j \in$ $\{d, i, e\}$, i.e., one adjoint problem per physical density field.

\section{Acknowledgements}

G. A. da Silva and A. T. Beck kindly acknowledge financial support of this research project by the agencies CNPq (National Council for Research and Development), grant number 306373/2016-5, and FAPESP (São Paulo Research Foundation), grant number 2015/25199-0. O. Sigmund was support by the Villum Investigator Project InnoTop funded by the Villum Foundation.

\section{References}

[1] P. Duysinx, M. P. Bendsøe, Topology optimization of continuum structures with local stress constraints, International Journal for Numerical Methods in Engineering 43 (8) (1998) 1453-1478. doi:10.1002/(SICI)1097-0207(19981230)43:8;1453::AID-NME480;3.0.CO;2-2.

[2] P. Duysinx, O. Sigmund, New developments in handling stress constraints in optimal material distribution, in: 7th AIAA/USAF/NASA/ISSMO Symposium on Multidisciplinary Analysis and Optimization, 1998, pp. 1501-. doi:10.2514/6.1998-4906.

[3] E. A. Fancello, J. T. Pereira, Structural topology optimization considering material failure constraints and multiple load conditions, Latin American Journal of Solids and Structures 1 (1) (2003) 3-24.

[4] J. T. Pereira, E. A. Fancello, C. S. Barcellos, Topology optimization of continuum structures with material failure constraints, Structural and Multidisciplinary Optimization 26 (1) (2004) 50-66. doi:10.1007/s00158-003-0301-z.

[5] E. A. Fancello, Topology optimization for minimum mass design considering local failure constraints and contact boundary conditions, Structural and Multidisciplinary Optimization 32 (3) (2006) 229-240. doi:10.1007/s00158-006-0019-9.

[6] M. Bruggi, On an alternative approach to stress constraints relaxation in topology optimization, Structural and Multidisciplinary Optimization 36 (2) (2008) 125-141. doi:10.1007/s00158-007-0203-6.

[7] J. París, F. Navarrina, I. Colominas, M. Casteleiro, Topology optimization of continuum structures with local and global stress constraints, Structural and Multidisciplinary Optimization 39 (4) (2009) 419-437. doi:10.1007/s00158-008-0336-2.

[8] C. Le, J. Norato, T. Bruns, C. Ha, D. Tortorelli, Stress-based topology optimization for continua, Structural and Multidisciplinary Optimization 41 (4) (2010) 605-620. doi:10.1007/s00158-009-0440-y.

[9] S. Amstutz, A. A. Novotny, Topological optimization of structures subject to von mises stress constraints, Structural and Multidisciplinary Optimization 41 (3) (2010) 407-420. doi:10.1007/s00158-009-0425-X.

[10] S. Amstutz, A. Novotny, E. de Souza Neto, Topological derivative-based topology optimization of structures subject to drucker-prager stress constraints, Computer Methods in Applied Mechanics and Engineering 233-236 (2012) 123 - 136. doi:10.1016/j.cma.2012.04.004.

[11] M. Bruggi, P. Duysinx, Topology optimization for minimum weight with compliance and stress constraints, Structural and Multidisciplinary Optimization 46 (3) (2012) 369-384. doi:10.1007/s00158-012-0759-7. 
[12] Y. Luo, Z. Kang, Topology optimization of continuum structures with drucker-prager yield stress constraints, Computers \& Structures 90-91 (2012) 65 - 75. doi:10.1016/j.compstruc.2011.10.008.

[13] Y. Luo, M. Y. Wang, Z. Kang, An enhanced aggregation method for topology optimization with local stress constraints, Computer Methods in Applied Mechanics and Engineering 254 (2013) 31 - 41. doi:10.1016/j.cma.2012.10.019.

[14] E. Holmberg, B. Torstenfelt, A. Klarbring, Stress constrained topology optimization, Structural and Multidisciplinary Optimization 48 (1) (2013) 33-47. doi:10.1007/s00158-012-0880-7.

[15] H. Emmendoerfer, E. A. Fancello, A level set approach for topology optimization with local stress constraints, International Journal for Numerical Methods in Engineering 99 (2) (2014) 129-156. doi:10.1002/nme.4676.

[16] H. Emmendoerfer, E. A. Fancello, Topology optimization with local stress constraint based on level set evolution via reactiondiffusion, Computer Methods in Applied Mechanics and Engineering 305 (2016) 62 - 88. doi:10.1016/j.cma.2016.02.024.

[17] H. Svärd, Interior value extrapolation: a new method for stress evaluation during topology optimization, Structural and Multidisciplinary Optimization 51 (3) (2015) 613-629. doi:10.1007/s00158-014-1171-2.

[18] D. M. De Leon, J. Alexandersen, J. S. O. Fonseca, O. Sigmund, Stress-constrained topology optimization for compliant mechanism design, Structural and Multidisciplinary Optimization 52 (5) (2015) 929-943. doi:10.1007/s00158-015-1279-z.

[19] C. Kiyono, S. Vatanabe, E. Silva, J. Reddy, A new multi-p-norm formulation approach for stress-based topology optimization design, Composite Structures 156 (2016) 10 - 19. doi:10.1016/j.compstruct.2016.05.058.

[20] M. Bruggi, Topology optimization with mixed finite elements on regular grids, Computer Methods in Applied Mechanics and Engineering 305 (2016) 133 - 153. doi:https://doi.org/10.1016/j.cma.2016.03.010.

[21] S. Zhang, A. L. Gain, J. A. Norato, Stress-based topology optimization with discrete geometric components, Computer Methods in Applied Mechanics and Engineering 325 (2017) 1 - 21. doi:https://doi.org/10.1016/j.cma.2017.06.025.

[22] R. Picelli, S. Townsend, C. Brampton, J. Norato, H. Kim, Stress-based shape and topology optimization with the level set method, Computer Methods in Applied Mechanics and Engineering 329 (2018) 1 - 23. doi:https://doi.org/10.1016/j.cma.2017.09.001.

[23] H. Lian, A. N. Christiansen, D. A. Tortorelli, O. Sigmund, N. Aage, Combined shape and topology optimization for minimization of maximal von mises stress, Structural and Multidisciplinary Optimization 55 (5) (2017) 1541-1557. doi:10.1007/s00158-017-1656-X.

[24] A. de Assis Pereira, E. L. Cardoso, On the influence of local and global stress constraint and filtering radius on the design of hinge-free compliant mechanisms, Structural and Multidisciplinary Optimizationdoi:10.1007/s00158-018-1915-5.

[25] G. A. da Silva, E. L. Cardoso, Stress-based topology optimization of continuum structures under uncertainties, Computer Methods in Applied Mechanics and Engineering 313 (2017) 647 - 672. doi:10.1016/j.cma.2016.09.049.

[26] G. A. da Silva, A. T. Beck, E. L. Cardoso, Topology optimization of continuum structures with stress constraints and uncertainties in loading, International Journal for Numerical Methods in Engineering 113 (1) (2018) 153-178. doi:10.1002/nme.5607.

[27] G. A. da Silva, A. T. Beck, Reliability-based topology optimization of continuum structures subject to local stress constraints, Structural and Multidisciplinary Optimization 57 (6) (2018) 2339-2355. doi:10.1007/s00158-017-1865-3.

[28] Y. Luo, M. Zhou, M. Y. Wang, Z. Deng, Reliability based topology optimization for continuum structures with local failure constraints, Computers \& Structures 143 (2014) 73 - 84. doi:10.1016/j.compstruc.2014.07.009.

[29] E. Holmberg, C.-J. Thore, A. Klarbring, Game theory approach to robust topology optimization with uncertain loading, Structural and Multidisciplinary Optimization (2016) 1-15doi:10.1007/s00158-016-1548-5.

[30] C.-J. Thore, E. Holmberg, A. Klarbring, A general framework for robust topology optimization under load-uncertainty including stress constraints, Computer Methods in Applied Mechanics and Engineering 319 (2017) 1 - 18. doi:10.1016/j.cma.2017.02.015.

[31] R. dosSantos, A. Torii, A. Novotny, Reliability-based topology optimization of structures under stress constraints, International Journal for 
Numerical Methods in Engineering 114 (6) (2018) 660-674. doi:10.1002/nme.5760.

[32] I. Elishakoff, M. Ohsaki, Optimization and Anti-Optimization of Structures Under Uncertainty, Imperial College Press, London, 2010.

[33] R. E. Melchers, A. T. Beck, Structural Reliability Analysis and Prediction, 3rd Edition, Wiley, 2018.

[34] J. Tu, K. K. Choi, Y. H. Park, A new study on reliability-based design optimization, Journal of Mechanical Design 121 (4) (1999) 557-564. doi:10.1115/1.2829499.

[35] H.-G. Beyer, B. Sendhoff, Robust optimization a comprehensive survey, Computer Methods in Applied Mechanics and Engineering 196 (3334) (2007) 3190 - 3218. doi:10.1016/j.cma.2007.03.003.

[36] O. Sigmund, Manufacturing tolerant topology optimization, Acta Mechanica Sinica 25 (2) (2009) 227-239. doi:10.1007/s10409-009-0240-z.

[37] F. Wang, B. S. Lazarov, O. Sigmund, On projection methods, convergence and robust formulations in topology optimization, Structural and Multidisciplinary Optimization 43 (6) (2011) 767-784. doi:10.1007/s00158-010-0602-y.

[38] O. Sigmund, K. Maute, Topology optimization approaches, Structural and Multidisciplinary Optimization 48 (6) (2013) $1031-1055$. doi:10.1007/s00158-013-0978-6.

[39] K.-J. Bathe, Finite Element Procedures, Prentice Hall, Upper Sadle River, New Jersey, 1996.

[40] O. Sigmund, Morphology-based black and white filters for topology optimization, Structural and Multidisciplinary Optimization 33 (4-5) (2007) 401-424. doi:10.1007/s00158-006-0087-x.

[41] S. Amstutz, Connections between topological sensitivity analysis and material interpolation schemes in topology optimization, Structural and Multidisciplinary Optimization 43 (6) (2011) 755-765. doi:10.1007/s00158-010-0607-6.

[42] G. D. Cheng, X. Guo, $\varepsilon$-relaxed approach in structural topology optimization, Structural Optimization (1997) 258266doi:10.1007/BF01197454.

[43] J. K. Guest, J. H. Prvost, T. Belytschko, Achieving minimum length scale in topology optimization using nodal design variables and projection functions, International Journal for Numerical Methods in Engineering 61 (2) (2004) 238-254. doi:10.1002/nme.1064.

[44] E. Birgin, J. Martínez, Practical Augmented Lagrangian Methods for Constrained Optimization, Society for Industrial and Applied Mathematics, Philadelphia, PA, 2014. doi:10.1137/1.9781611973365.

[45] S. S. Rao, Engineering Optimization: Theory and Practice (Fourth Edition), Wiley, New Jersey, 2009.

[46] S. J. Hollister, B. A. Riemer, Digital-image-based finite element analysis for bone microstructure using conjugate gradient and gaussian filter techniques, in: Proc.SPIE, Vol. 2035, 1993, pp. 95-106. doi:10.1117/12.146616.

[47] R. Guldberg, S. Hollister, G. Charras, The accuracy of digital image-based finite element models, Journal of Biomechanical Engineering 120 (2) (1998) 289-295. doi:10.1115/1.2798314.

[48] G. Charras, R. Guldberg, Improving the local solution accuracy of large-scale digital image-based finite element analyses, Journal of Biomechanics 33 (2000) 255 - 259. doi:10.1016/S0021-9290(99)00141-4.

[49] A. Doitrand, C. Fagiano, F.-X. Irisarri, M. Hirsekorn, Comparison between voxel and consistent meso-scale models of woven composites, Composites Part A: Applied Science and Manufacturing 73 (2015) 143 - 154. doi:https://doi.org/10.1016/j.compositesa.2015.02.022.

[50] G. Fang, B. E. Said, D. Ivanov, S. R. Hallett, Smoothing artificial stress concentrations in voxel-based models of textile composites, Composites Part A: Applied Science and Manufacturing 80 (2016) 270 - 284. doi:https://doi.org/10.1016/j.compositesa.2015.10.025.

[51] W. Pilkey, Peterson's stress concentration factors, John Wiley \& sons, New York, 2007. doi:10.1002/9780470172674.

[52] M. Stolpe, K. Svanberg, An alternative interpolation scheme for minimum compliance topology optimization, Structural and Multidisciplinary Optimization 22 (2) (2001) 116-124. doi:10.1007/s001580100129.

[53] A. Clausen, E. Andreassen, On filter boundary conditions in topology optimization, Structural and Multidisciplinary Optimization 56 (5) (2017) 1147-1155. doi:10.1007/s00158-017-1709-1. 
[54] G. Christophe, R. Jean-Francois, Gmsh: A 3-d finite element mesh generator with built-in pre- and post-processing facilities, International Journal for Numerical Methods in Engineering 79 (11) (2009) 1309-1331. doi:10.1002/nme.2579. 